\title{
Unified theory of quantized electrons, phonons, and photons out of equilibrium: A simplified $a b$ initio approach based on the generalized Baym-Kadanoff ansatz
}

\author{
Pedro Miguel M. C. de Melo ${ }^{1,2}$ and Andrea Marini ${ }^{1,3}$ \\ ${ }^{1}$ Istituto di Struttura della Materia of the National Research Council, Via Salaria Km 29.3,I-00016 Monterotondo Stazione, Italy \\ ${ }^{2}$ CFisUC, Department of Physics, University of Coimbra, Rua Larga, 3004-516 Coimbra, Portugal \\ ${ }^{3}$ European Theoretical Spectroscopy Facilities (ETSF)
}

(Received 23 December 2015; revised manuscript received 26 February 2016; published 4 April 2016)

\begin{abstract}
We present a full $a b$ initio description of the coupled out-of-equilibrium dynamics of photons, phonons, and electrons. In the present approach, the quantized nature of the electromagnetic field as well as of the nuclear oscillations is fully taken into account. The result is a set of integrodifferential equations, written on the Keldysh contour, for the Green's functions of electrons, phonons, and photons where the different kinds of interactions are merged together. We then concentrate on the electronic dynamics in order to reduce the problem to a computationally feasible approach. By using the generalized Baym-Kadanoff ansatz and the completed collision approximation, we introduce a series of efficient but controllable approximations. In this way, we reduce all equations to a set of decoupled equations for the density matrix that describe all kinds of static and dynamical correlations. The final result is a coherent, general, and inclusive scheme to calculate several physical quantities: carrier dynamics, transient photoabsorption, and light emission, all of which include, at the same time, electron-electron, electron-phonon, and electron-photon interactions. We further discuss how all these observables can be easily calculated within the present scheme using a fully atomistic ab initio approach.
\end{abstract}

DOI: 10.1103/PhysRevB.93.155102

\section{INTRODUCTION}

The impressive progresses in ultrafast and ultrastrong laser-pulse technology have paved the way to the modern nonequilibrium (NEQ) attosecond spectroscopies [1-5]. Unlike conventional spectroscopies, the sample is driven away from equilibrium by a strong laser pulse (the pump) and then probed with a weaker field (the probe). This second field is used to monitor a wealth of physical properties of the material in order to disclose the complex properties of the excited system through the different phases of the real-time evolution [6,7]. Indeed, experiments are carried out using pump pulses with frequency in the infrared-ultraviolet range and ultrashort probe pulses (down to a few hundreds of attoseconds). By varying the delay between the pump and the probe pulses, one can monitor the excited-state dynamics in a wide energy and time range.

The elemental processes that are induced by the perturbation with a strong laser field and studied in this work are schematically represented in Fig. 1. The external laser pulse first excites a certain density of carriers from the valence to the conduction bands. The duration of this process is directly controlled by the duration of the laser field. In addition, the density of carriers is dictated by the intensity of the laser that also controls the amount of energy transferred to electrons and holes [6-12].

Already during this first step of the whole dynamics, static and coherent correlation effects play a crucial role. As an example, it is well known that the light absorption process is accompanied by the formation of excitonic states [13]. These are bound electron-hole pairs created by the initial laser excitation. The electron-hole attraction is described by a screened Coulomb interaction. Nevertheless, at this stage the quantization of the electromagnetic field is not requested and, indeed, in studying simple optical absorption the simpler classic treatment is commonly adopted $[13,14]$.
After the photoexcitation, the carriers will relax by dissipating and transferring energy among themselves and to the lattice. During this first step of the dynamics (that can be as long as few picoseconds), the ensemble of electrons interact via repeated collisions mediated by the Coulomb $(\mathrm{e}-\mathrm{e})$ and the electron-phonon $(\mathrm{e}-\mathrm{p})$ interactions. Indeed, the complex interaction of the carriers with the lattice imposes the quantization of the atomic oscillations in the form of phonon modes. These then enter naturally in the dynamics and must be included in a coherent framework together with excitonic effects. This already represents a first important and challenging aspect in the description of out-of-equilibrium processes.

The last step in the dynamics of the photoexcited carriers is the recombination with the consequent spontaneous light emission. This process is entirely a quantistic phenomenon and requires the quantization of the electromagnetic field. As a consequence, it is evident that a comprehensive description of all phases of the dynamics following a photoexcitation event requires the simultaneous quantistic treatment of electron, phonons, and photons. This is well beyond the state of the art and it represents the goal of this work.

In a typical pump and probe experiment (P\&p), a weaker and perturbative second laser pulse is used to probe the system at any time between the processes (a) and (c) of Fig. 1. A wealth of time-dependent observables are then measured experimentally. Examples are the change in the absorption of the probe induced by the pump (transient absorption) [15-17], the time-dependent light-emission spectrum [18], the time-dependent photoelectron spectrum [19,20].

The key point is that all these observables are inherently connected, as they are produced from the same elemental dynamics of the system, although from different perspectives. From a theoretical point of view, the device of a coherent description of the excited state of the materials has followed 


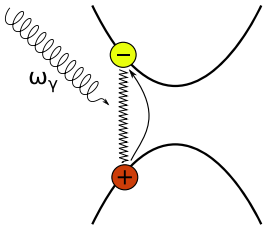

(a)

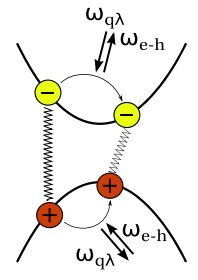

(b)

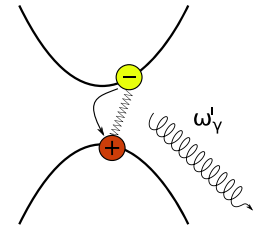

(c)
FIG. 1. Schematic representation of the different processes induced by the interaction of a material with a short and intense laser pulse. The action of the laser creates electron-hole pairs [process (a)]. These pairs interact through the screened Coulomb interaction (zigzag line) creating transient excitonic states. After the photoexcitation, the carriers undergo repeated collisions with other carriers and with the lattice [process (b)]. During these processes, phonons and electron-hole pairs are emitted and/or absorbed. Finally, after picoseconds to microseconds, the excited carriers eventually relax to the ground-state emitting photons [process (c)]. $\omega_{\gamma}$ and $\omega_{\gamma}^{\prime}$ are photon energies, $\omega_{\mathbf{q} \lambda}$ is a phonon energy, and $\omega_{\mathrm{e}-\mathrm{h}}$ represents electron-hole pair energy.

several distinct and often fragmented and uncorrelated paths. The most up-to-date scheme to calculate and predict the ground- and excited-state properties of a wide range of materials is based on the merging of density functional theory (DFT) [21] with many-body perturbation theory (MBPT) [13]. DFT is a broadly used ab initio ground-state theory, that allows to calculate exactly the electronic density and total energy without adjustable parameters. The merging of DFT with perturbation theory gives the so-called density functional perturbation theory $[22,23]$ (DFPT). The DFPT is a powerful computational tool for the direct treatment of phonons. However, the DFT computation of excited electronic states properties, like the band-gap energies, is a known problematic topic [21]. As a result, MBPT is nowadays the preferred alternative to DFT for that purpose. It is based on the accurate treatment of correlation effects by means of the Green's function formalism. MBPT is formally correct and leads to a close agreement with experiment [24], but is extremely computationally demanding. A natural way to solve this issue is to merge the quick DFT calculation with the accurate MBPT one. The latter method is often referred to as $a b$ initio many-body perturbation theory [13] (ai-MBPT). In this method, DFT provides a suitable single-particle basis for the MBPT scheme. This method has been applied successfully, for example, to correct the well-known band-gap underestimation problem of DFT [25].

Therefore, as far as equilibrium properties are concerned, the theoretical and methodological developments have constantly contributed to create a consistent and efficient environment that can now count on a number of well-established standards and codes.

The situation in the out-of-equilibrium case is rather different. Indeed, the standard tools of equilibrium MBPT cannot be applied and one has to switch to more advanced nonequilibrium Green's function (NEGF) techniques. From a purely theoretical point of view, the NEGF theory has been extensively studied and reviewed in many books [9-12]. Nevertheless, its development has been mainly confined to simple models or specific models suitable to interpret specific properties. A merging with DFT, in the NEGF case, is still at the very beginning and an inclusive approach is lacking. As a main consequence, there are not standard numerical tools that can be used even by nonexperts or experimentalists to support their observations. This is the main motivation of this work.

The NEGF theory is indeed, as far as the electronelectron (e-e) interaction is concerned, at an excellent level of development. By using the Keldysh contour formalism [26], we can obtain the Baym-Kadanoff equations (BKE) that govern the electronic motion. The fundamental ingredient of the BKE is the self-energy, which embodies all the information on the many-particle interaction. The self-energy can be calculated from single-particle quantities [26]. The BKE can also be reduced to a Boltzmann-type equation in the Markovian limit [26].

The e-p interaction has been widely studied both at the and out of the equilibrium. In the first case, the MBPT approach has been applied and reviewed in Ref. [27]. In this case, the nuclear effects are included by deriving a full set of self-consistent equations for the screened interaction and the self-energy operator. Also, the merging of MBPT and DFT has been studied $[28,29]$ and applied [30-34] extensively. In the out-of-equilibrium case, the theory is well known as well and it leads to a combined description of both the electronic and phononic dynamics $[10,35,36]$. In this case, the merging with DFT has been only very recently introduced within the simplified Markovian limit [37] or in the more general scheme based on the generalized Baym-Kadanoff ansatz (GBKA) and on the completed collision approximation (CCA) [20,38].

It is crucial to note, at this point, that in all the above cases the electron-photon $(\mathrm{e}-\gamma)$ interaction is neglected and the electromagnetic interaction is treated classically. As we will see shortly, this approach is suitable unless we are not interested in the long-time carrier dynamics and in the transient light-emission spectrum.

Indeed, another family of theoretical studies is connected to the bridging of the very general theories based on the NEGF with the actual experiments that are performed in a typical P\&p setup. The case of the carrier dynamics is the most natural and easy to introduce as it is a simple by product of the BKE. Once that GBKA and the CCA are applied, the equation of motion for the electronic occupations is obtained [20,35-38].

The time-resolved light absorption of the probe field has been studied within the NEGF formalism in Ref. [39]. Starting from the BKE and introducing an adiabatic approximation, an equation of motion for the linear response function is derived and shown to reduce to the well-known Bethe-Salpeter equation (BSE) in the absence of the pump field or when this is weak enough to be possible to apply the low-intensity regime approximation (LIA). Both the LIA and the adiabatic ansatz will be introduced and extensively used in this work.

In order to study photoluminescence (PL), the inclusion of the e-e and e-p interactions is not enough. Indeed, light emission is possible only when also the electromagnetic field is quantized and it appears as a evolving term in the BKE. As in the e-e and e-p cases also the inclusion of the e- $\gamma$ interaction has been studied [40-42]. In these works, the theory is bridged with the BSE but the e-p interaction, and therefore the photoexcited carriers relaxation, is neglected. 
Alternatively, e-p interaction has been included $[41,43,44]$ but, in this case, neglecting the e-e interaction and, therefore, the formation of excitonic states.

A field that has been developed in parallel to MBPT is the one based on DFT. In DFT, the most used tool is the time-dependent Kohn-Sham equations [21] that have been applied to study out-of-equilibrium regimes $[45,46]$. DFT provides an alternative and powerful tool where, however, the description of e-e and e-p interactions is quite problematic. Indeed, DFT has been mainly applied to low-dimensional systems [47] or to study very short-time effects [48] where relaxation, dissipation, and formation of excitonic states can be, within specific approximations, neglected. Quantized electromagnetic fields have been recently introduced within DFT [49].

This short introduction proves that the development of a theory of quantized electrons, phonons, and photons out of equilibrium is fragmented in several, often uncorrelated, paths. In addition, these approaches often use very different schemes and approximations, often difficult to merge together. In addition, the complexity of all these theories has prevented their application to realistic materials at a level of accuracy comparable with the $a i$-MBPT approach. This has deprived the theory of its potential predictive power. Also because, in general, P\&p experiments are used to measure changes in specific observables, which are often very small quantities, therefore making the accuracy of the theory very important. The merging with DFT has been only recently introduced and it is still at the initial stages of its development [14,37,38,50].

In this paper, we present a full description of an interacting system, thus including electrons, photons, and phonons, out of equilibrium. We start from the many-body Hamiltonian and by following a step-by-step mathematical procedure based on NEGF theory we derive the complete BKE including coherently the three interactions: e-e, e-p, and e- $\gamma$. In this way, we go beyond the state-of-the-art knowledge preparing the field for the merging with DFT. Indeed, in order to do so, we discuss key physical regimes (the low-intensity and adiabatic regimes) from which we derive key approximations. By using these approximations, the overall complexity of the equations will be greatly simplified and suitable to be merged with DFT in a computationally feasible approach to out-of-equilibrium phenomena.

The structure of the paper is as follows. We will first introduce the problem, the Hamiltonian (Sec. II), and the interaction terms (Sec. II A). By extending the seminal works of Ref. [27] on the electron-phonon interaction and of Refs. [26,40,42,51] on the electron-photon interaction, we will introduce in Sec. III A the equation of motion for the Green's function, defined in Sec. III, on the Keldysh contour. We will discuss extensively the longitudinal and transverse response functions (Sec. III C) and vertex functions (Sec. III D). Although we derive a full consistent set of equations for all the different parts entering the scattering process, here we will focus on the electronic dynamics. The equations of motion relative to the other particles (photons and phonons) will be introduced in a future work. In Sec. IV, we will introduce the $G W$ approximation and, in Sec. V, this approximation will be used to close the dynamics in the space of the single-time density matrices by means of the GBKA (Sec. V). In addition, we will investigate further simplifications obtained by treating the memory effects within the CCA (Sec. VB). Thanks to these crucial approximations we will arrive to a closed equation, in Sec. VI, that will allow us to derive simple but efficient equations to describe the carrier dynamics (Sec. VI B), the transient optical absorption (Sec. VIC), and, finally, the light-emission spectrum (Sec. VID). We will conclude this work by rewriting the theory in the frequency domain by introducing an adiabatic ansatz (Sec. VII) and by discussing the merging with the $a b$ initio methods (Sec. VIII).

\section{BARE HAMILTONIAN AND THE INTERACTION TERMS}

We start with the nonrelativistic Hamiltonian of a system of interacting electrons moving under the action of an external electromagnetic field and of the internal electron-nucleus interaction:

$$
\begin{aligned}
\hat{H} & =\hat{H}_{0}+\hat{H}_{\mathrm{int}}, \\
\hat{H}_{0} & =\sum_{i} h\left(\hat{\mathbf{r}}_{i}\right)+\hat{T}_{\mathrm{n}}+\hat{H}_{\gamma}+\hat{H}_{\mathrm{n}-\mathrm{n}}, \\
\hat{H}_{\mathrm{int}} & =\hat{H}_{\mathrm{e}-\mathrm{e}}+\hat{H}_{\mathrm{e}-\mathrm{n}}+\hat{H}_{\mathrm{e}-\gamma} .
\end{aligned}
$$

Equation (1) includes terms describing the single-particle dynamics $\left(\hat{T}_{\mathrm{e}}, \hat{T}_{\mathrm{n}}\right.$, and $\left.\hat{H}_{\gamma}\right)$ and interaction terms due to the mutual interaction of electrons, nuclei, and photons $\left(\hat{H}_{\mathrm{n}-\mathrm{n}}, \hat{H}_{\mathrm{e}-\mathrm{n}}, \hat{H}_{\mathrm{e}-\mathrm{e}}\right.$, and $\left.\hat{H}_{\mathrm{e}-\gamma}\right)$. In the above equation, $h(\mathbf{r})$ represents the singleparticle operator and it is summed over the electronic positions $\mathbf{r}_{i}$.

Depending on the choice of the noninteracting part of the Hamiltonian, $h$ can include, aside from the kinetic part, some kind of initial correlation in the form of a mean-field potential. This is an essential ingredient in the merging of many-body techniques with $a b$ initio methods.

The electronic and nuclear kinetic parts are

$$
\begin{aligned}
& \hat{T}_{\mathrm{e}}=-\frac{1}{2} \int d^{3} r \hat{\psi}^{\dagger}(\mathbf{r}) \nabla^{2} \hat{\psi}(\mathbf{r}), \\
& \hat{T}_{\mathrm{n}}=-\sum_{\mathbf{R}} \frac{\nabla_{\mathbf{R}}^{2}}{2 M_{\mathbf{R}}},
\end{aligned}
$$

with $\mathbf{R}$ the generic position of the nucleus with mass $M_{\mathbf{R}}$. In Eq. (2), $\hat{\psi}(\mathbf{r})$ and $\hat{\psi}^{\dagger}(\mathbf{r})$ are, respectively, the electron creation and annihilation operators in the Schrödinger's picture. The spinorial degrees of freedom are not considered here to keep the notation as simple as possible. The extension of the present theory to include their effect can be done starting from Pauli's equation and using the minimal coupling transformation. We also use the convention of representing vectors with a bold symbol (like A) and tensors using a double arrow overscript (like $\overleftrightarrow{\mathcal{D}}$ ). Through this work, band indices will be represented by a Latin subscript $(i, j, \ldots)$, while Cartesian directions and branch indices will be denoted by Greek labels $(\alpha, \beta, \ldots)$.

$\hat{H}_{\gamma}$ is the noninteracting Hamiltonian for the transverse photons

$$
\hat{H}_{\gamma}=\sum_{\mathbf{q}, \lambda} \omega_{\mathbf{q}} \hat{d}_{\mathbf{q}, \lambda}^{\dagger} \hat{d}_{\mathbf{q}, \lambda}
$$


with $\mathbf{q}$ and $\omega_{\mathbf{q}}$ the photon's momentum and energy, $\lambda$ its polarization, and $\hat{d}_{\mathbf{q}, \lambda}^{\dagger}$ and $\hat{d}_{\mathbf{q}, \lambda}$ the creation and annihilation operators, respectively (also in Schrödinger's picture).

The first group of interaction terms describes the nuclearnuclear and e-e interactions. Those do not make the different subspaces of electron, nuclei, and photons interact but build up internal (purely electronic and nuclear) correlation effects:

$$
\hat{H}_{\mathrm{e}-\mathrm{e}}=\frac{1}{2} \int d^{3} r d^{3} r^{\prime} \hat{\psi}^{\dagger}(\mathbf{r}) \hat{\psi}^{\dagger}\left(\mathbf{r}^{\prime}\right) v\left(\mathbf{r}-\mathbf{r}^{\prime}\right) \hat{\psi}\left(\mathbf{r}^{\prime}\right) \hat{\psi}(\mathbf{r}),
$$

with $v\left(\mathbf{r}-\mathbf{r}^{\prime}\right)$ the bare Coulomb potential. The nucleusnucleus interaction reads as

$$
\tilde{H}_{\mathrm{n}-\mathrm{n}}=\frac{1}{2} \sum_{\mathbf{R}, \mathbf{R}^{\prime}}^{\prime} Z_{\mathbf{R}} Z_{\mathbf{R}^{\prime}} v\left(\mathbf{R}-\mathbf{R}^{\prime}\right),
$$

with $\sum_{i j}^{\prime}=\sum_{i \neq j}$ and $Z_{\mathbf{R}}$ the nucleus atomic number.

The e-p interaction $\hat{H}_{\mathrm{e}-\gamma}$ is given by

$$
\hat{H}_{\mathrm{e}-\gamma}=-\frac{1}{c} \int d^{3} r \hat{\mathbf{A}}(\mathbf{r}) \cdot \hat{\mathbf{J}}(\mathbf{r})+\frac{1}{2 c^{2}} \int d^{3} r \hat{\rho}(\mathbf{r}) \hat{\mathbf{A}}^{2}(\mathbf{r}) .
$$

Here, the paramagnetic electronic current and the density are defined as

$$
\begin{aligned}
& \hat{\mathbf{J}}(\mathbf{r})=\frac{1}{2 \mathrm{i}}\left[\hat{\psi}^{\dagger}(\mathbf{r}) \nabla \hat{\psi}(\mathbf{r})-\text { c.c. }\right], \\
& \hat{\rho}(\mathbf{r})=\hat{\psi}^{\dagger}(\mathbf{r}) \hat{\psi}(\mathbf{r}) .
\end{aligned}
$$

We work in the second quantization formalism and introduce a suitable single-particle basis with orthonormal wave functions $\left\{\varphi_{i}(\mathbf{r})\right\}$. Then, the creation and annihilation field operators $\hat{\psi}^{\dagger}(\mathbf{r})$ and $\hat{\psi}(\mathbf{r})$ for a particle at position $\mathbf{r}$ in space are expanded according to

$$
\hat{\psi}(\mathbf{r})=\sum_{i} \varphi_{i}(\mathbf{r}) \hat{c}_{i}
$$

The one-particle density-matrix operator takes the form

$$
\hat{\rho}\left(\mathbf{r}, \mathbf{r}^{\prime}\right)=\hat{\psi}^{\dagger}(\mathbf{r}) \hat{\psi}\left(\mathbf{r}^{\prime}\right)=\sum_{i j} \varphi_{i}^{*}(\mathbf{r}) \varphi_{j}\left(\mathbf{r}^{\prime}\right) \hat{\rho}_{j i},
$$

with $\hat{\rho}_{j i}=\hat{c}_{i}^{\dagger} \hat{c}_{j}$.

The first important step is the quantization of the electromagnetic field that, as it will be clear shortly, will appear as an explicit ingredient of the time evolution. We start by rewriting the vector potential of the electromagnetic radiation in terms of photon creation and annihilation operators

$$
\begin{aligned}
\hat{\mathbf{A}}(\mathbf{r})= & \sum_{\mathbf{G}, \mathbf{q}, \lambda}\left(\frac{2 \pi c^{2}}{\omega_{\mathbf{q}+\mathbf{G}} \Omega}\right)^{\frac{1}{2}}\left[\hat{d}_{\mathbf{q}+\mathbf{G}, \lambda} e^{i(\mathbf{q}+\mathbf{G}) \cdot \mathbf{r}}\right. \\
& \left.+\hat{d}_{\mathbf{q}+\mathbf{G}, \lambda}^{\dagger} e^{-i(\mathbf{q}+\mathbf{G}) \cdot \mathbf{r}}\right] \mathbf{e}_{\lambda}(\mathbf{q}+\mathbf{G}),
\end{aligned}
$$

with $\Omega$ being the volume of the lattice and $\mathbf{e}_{\lambda}(\mathbf{q}+\mathbf{G})$ the polarization vectors orthogonal to the photon's momentum $\mathbf{q}+\mathbf{G}$.

We aim at describing any kind of system by using a supercell approach. This means that the periodic part of the system (if any) is represented by a unit cell of volume $\Omega_{s}$ containing $N$ momenta $\mathbf{q}\left(\Omega \equiv N \Omega_{s}\right)$. This unit cell is periodically repeated displaced of a generic vector $\mathbf{G}$ of the reciprocal lattice. In the case of isolated systems $N=1$ and $\Omega_{s}$ is chosen large enough to avoid spurious interactions.

The e-p interaction arises from the term $\hat{H}_{\mathrm{e}-\mathrm{n}}$ where the nuclear and electronic densities are coupled via the Coulomb interaction

$$
\hat{H}_{\mathrm{e}-\mathrm{n}}=-\int d \mathbf{r} d \mathbf{R} \frac{\hat{\rho}(\mathbf{r}) \hat{N}(\mathbf{R})}{|\mathbf{r}-\mathbf{R}|} .
$$

$\hat{N}(\mathbf{R})$ is the nuclear density operator that we take as the counterpart of the electronic case. The actual definition of the nuclear density is a delicate issue that has been already discussed in Ref. [27].

\section{A. Coupling to the external perturbations}

The Hamiltonian $H$ describes the complete dynamics of the coupled systems of electrons, photons, and phonons. In order to rewrite this dynamics in the form of equations of motion for the corresponding Green's functions, we can use two equivalent paths. One is based on the standard diagrammatic technique [52] which constructs approximations for the different terms of the theory by using a geometrical and graphical approach. An alternative approach, that we follow here, is based instead on the equation-of-motion approach [53]. This method leads, both in the equilibrium and the out-of-equilibrium regimes, to a closed set of integrodifferential equations that at the equilibrium are known as Hedin's equations [54].

In order to extend these equations to account for the correlated dynamics of electrons, photons, and phonons in an out-of-equilibrium context, we start by discussing some key aspects of the purely electronic case. In the equation-ofmotion approach, the Hamiltonian is perturbed with a fictitious time-dependent term

$$
\hat{H}(t)=\hat{H}+\hat{H}_{\text {ext }}(t) .
$$

In the original derivation of Hedin's equations [53], $\hat{H}_{\mathrm{ext}}(t)$ describes the coupling of the electronic charge with an external fictitious field $\phi_{\mathrm{ext}}(\mathbf{r}, t)$ that, at the end of the derivation, is set to zero:

$$
\hat{H}_{\mathrm{ext}}(t)=\int d^{3} r \phi_{\mathrm{ext}}(\mathbf{r}, t) \hat{\rho}(\mathbf{r}, t) .
$$

Now, the problem is that this perturbation cannot be used in the present case where we want to describe a quantized electromagnetic field. The reason is that the vector potential is now quantized and it cannot be set to zero at the end of the calculations. This is connected to the well-known existence of a vacuum energy of the electromagnetic field.

To solve this problem, we follow a different path. We notice that the external potential $\phi_{\text {ext }}(\mathbf{r}, t)$ is solution of the Poisson equation

$$
\nabla^{2} \phi_{\mathrm{ext}}(\mathbf{r}, t)=-4 \pi \rho_{\mathrm{ext}}(\mathbf{r}, t) .
$$

The solution of Eq. (14) can be rewritten in integral form

$$
\phi_{\mathrm{ext}}(\mathbf{r}, t)=\int d^{3} r^{\prime} v\left(\mathbf{r}-\mathbf{r}^{\prime}\right) \rho_{\mathrm{ext}}\left(\mathbf{r}^{\prime}, t\right),
$$

that, plugged into Eq. (13), yields

$$
\hat{H}_{\text {ext }}(t)=\int d^{3} r \hat{\phi}(\mathbf{r}) \rho_{\mathrm{ext}}(\mathbf{r}, t),
$$

with $\rho_{\text {ext }}$ the inhomogeneous part of Eq. (14). 
By comparing Eq. (13) with (16), we notice that, now, the potential can be quantized and the external charge sent to zero. As it will be clear in the following, these two procedures are equivalent, as far as the solely electronic limit is concerned.

Another important aspect is that we can now couple the external test charge to the potential generated by both the nuclear and the electronic charges:

$$
\begin{aligned}
\hat{\phi}(\mathbf{r}, t) & =\int d^{3} r^{\prime} v\left(\mathbf{r}-\mathbf{r}^{\prime}\right)\left[\hat{\rho}\left(\mathbf{r}^{\prime}\right)-\hat{N}\left(\mathbf{r}^{\prime}\right)\right] \\
& =\int d^{3} r^{\prime} v\left(\mathbf{r}-\mathbf{r}^{\prime}\right) \hat{n}\left(\mathbf{r}^{\prime}\right),
\end{aligned}
$$

where $\hat{n}=\hat{\rho}-\hat{N}$ is the total internal density of the system, since now we have to account for the quantized nuclei as well. By following Ref. [27] we introduce an external perturbation of the nuclear density $N_{\text {ext }}(\mathbf{R}, t)$, that is coupled, via Eq. (15), to the electric potential generated by the nuclei $\hat{V}_{\mathrm{n}}(\mathbf{r}, t)$.

A similar procedure can be applied to the perturbation induced by an external vector potential by using the equation of motion

$$
\left(\frac{1}{c^{2}} \partial_{t}^{2}-\nabla^{2}\right) \mathbf{A}_{\text {ext }}(\mathbf{r}, t)=\frac{4 \pi}{c} \mathbf{J}_{\text {ext }}(\mathbf{r}, t) .
$$

In this way, the total interaction part of the Hamiltonian looks like

$$
\begin{aligned}
\hat{H}_{\mathrm{ext}}(t)= & \int d^{3} r \hat{\phi}(\mathbf{r}, t) \rho_{\mathrm{ext}}(\mathbf{r}, t)-\frac{1}{c} \int d^{3} r \hat{\mathbf{A}}(\mathbf{r}) \cdot \mathbf{J}_{\mathrm{ext}}(\mathbf{r}, t) \\
& -\int d^{3} R \hat{V}_{\mathrm{n}}(\mathbf{R}) N_{\mathrm{ext}}(\mathbf{R}, t) .
\end{aligned}
$$

The last term in Eq. (19) describes the coupling with the nuclear motion. This was first introduced by Baym in Ref. [55] and also used in Ref. [27]. Here, we work with a fictitious external density in order to maintain coherence with the other perturbative terms. As it will be clear in the next sections, we use these external quantities to decouple the dynamics of electrons, nuclei, and photons. In Eq. (19) we have neglected the second term on the right-hand side of Eq. (6). The reason is that this term is multiplied by $1 / c^{2}$ and therefore, for a given order in the perturbative expansion, it leads to correction much smaller than those induced by the $\mathbf{A} \cdot \mathbf{J}$ term. From a diagrammatic point of view, the $\rho \mathbf{A}^{2}$ term is responsible of a series of diagrams well known and studied in the e-p problem [28], where they arise from the second-order e-p interaction. In the e- $\gamma$ case, however, these diagrams can be safely neglected.

\section{GREEN'S FUNCTIONS AND EQUATIONS OF MOTION ON THE KELDYSH CONTOUR}

The time evolution in nonequilibrium processes is more complicated than for equilibrium systems because it is not guaranteed that after an arbitrarily long enough time the system will return to the ground state. The extension of Hedin's equations to an out-of-equilibrium system can be done with recourse to a contour in the complex plane known as the Keldysh contour [10]. The contour runs as shown in Fig. 2 with the upper (positive) branch running from an instant $t_{0}$ where the system is assumed to be at equilibrium to an unknown state. The system is brought back to equilibrium via the lower

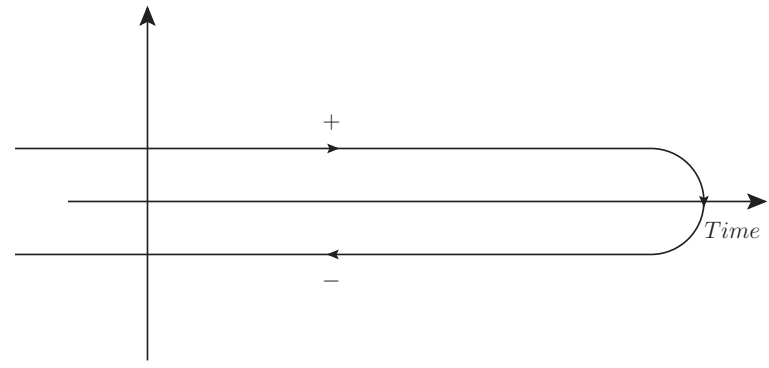

FIG. 2. The Keldysh contour. Any time index runs on the contour which defines a natural time ordering that only in the upper branch is equivalent to the ordering in the standard real-time axis.

(negative) branch and it is this mathematical description for the time evolution which permits us to have all the necessary rules to reconstruct an equation system similar to the original one of Hedin's [10].

Therefore, the Green's function $G(1,2)$ for nonequilibrium processes is defined on the Keldysh contour [11]

$$
G\left(1_{\alpha}, 2_{\beta}\right)=-i \beta \frac{\operatorname{Tr}\left\{\rho_{0} \mathrm{~T}_{C}\left[\hat{S}_{C} \hat{\psi}_{I}\left(1_{\alpha}\right) \hat{\psi}_{I}^{\dagger}\left(2_{\beta}\right)\right]\right\}}{\operatorname{Tr}\left\{\rho_{0} \hat{S}_{C}\right\}},
$$

where the subscript $I$ indicates that operators are in the interaction picture and subscripts $\alpha, \beta= \pm 1$ indicate the branch on the Keldysh contour where the time argument of the respective operator is located. The operator $\mathrm{T}_{C}$ is the contour time-ordering operator. In Eq. (20), we introduce the compact notation $1 \equiv\left(\mathbf{r}_{1}, t_{1}\right)$. Note that the time $t$ runs on the Keldysh contour.

The transition from the Heisenberg picture (where the electron operators are usually defined) to the interaction one is done via the following expression:

$$
\hat{O}\left(t_{\alpha}\right)=\hat{S}\left(-\infty, t_{\alpha}\right) \hat{O}_{I}\left(t_{\alpha}\right) \hat{S}\left(t_{\alpha},-\infty\right)
$$

where $\hat{O}$ is a generic operator and

$$
\hat{S}\left(t_{\alpha}, t_{\alpha}^{\prime}\right)=\mathrm{T}_{\alpha} \exp \left[-i \alpha \int_{t_{\alpha}^{\prime}}^{t_{\alpha}} d \tau \hat{H}_{\mathrm{ext}, I}^{\alpha}(\tau)\right] .
$$

Note that, in Eq. (22), the time arguments lie on a single time branch of the Keldysh contour. This implies that we can introduce a branch-specific evolution operator $\hat{S}_{\alpha}\left(t, t^{\prime}\right) \equiv \hat{S}\left(t_{\alpha}, t_{\alpha}^{\prime}\right)$. With this definition the overall time evolution operator $\hat{S}_{C}$ entering Eq. (20) can be rewritten as

$$
\hat{S}_{C}=\hat{S}_{-}(-\infty, \infty) \hat{S}_{+}(\infty,-\infty),
$$

and we have that the expectation value of $\hat{O}$ taken on the contour is

$$
\langle\hat{O}(1)\rangle_{C}=\frac{\operatorname{Tr}\left\{\rho_{0} \mathbf{T}_{C}\left[\hat{S}_{C} \hat{O}_{I}(1)\right]\right\}}{\operatorname{Tr}\left\{\rho_{0} \hat{S}_{C}\right\}} .
$$

The structure of Eq. (20) with respect to the position of the time arguments on the Keldysh contour defines the different kind of Green's functions:

$$
\begin{aligned}
& G\left(1_{+}, 2_{+}\right) \equiv G_{c}(1,2), \\
& G\left(1_{-}, 2_{+}\right) \equiv G^{<}(1,2), \\
& G\left(1_{+}, 2_{-}\right) \equiv G^{>}(1,2), \\
& G\left(1_{-}, 2_{-}\right) \equiv G_{\tilde{c}}(1,2) .
\end{aligned}
$$


In Eq. (25), $G_{c}$ is the time-ordered or causal Green's function, $G^{<}$and $G^{>}$are the lesser and greater Green's functions, and $G_{\tilde{c}}$ is the anti-time-ordered Green's function.

\section{A. Equation of motion for the Green's function on the Keldysh contour}

In order to obtain the equation of motion for $G$ we start from $i \partial_{t_{1}} G\left(1_{\alpha}, 2_{\beta}\right)$. From Eqs. (20) and (25) we get

$$
\begin{aligned}
\partial_{t_{1}} \mathrm{~T}_{C} & {\left[\hat{S}_{C} \hat{\psi}_{I}\left(1_{\alpha}\right) \hat{\psi}_{I}^{\dagger}\left(2_{\beta}\right)\right] } \\
= & \delta\left(1_{\alpha}-2_{\beta}\right) \mathrm{T}_{C}\left[\hat{S}_{C}\right]+\mathrm{T}_{C}\left[\hat{S}_{C} \partial_{t_{1}} \hat{\psi}_{I}\left(1_{\alpha}\right) \hat{\psi}_{I}^{\dagger}\left(2_{\beta}\right)\right] \\
& +i \alpha \mathrm{T}_{C}\left[\hat{S}_{C} \hat{H}_{\mathrm{ext}}\left(t_{1 \alpha}\right) \hat{\psi}_{I}\left(1_{\alpha}\right) \hat{\psi}_{I}^{\dagger}\left(2_{\beta}\right)\right] \\
& -i \alpha \mathrm{T}_{C}\left[\hat{S}_{C} \hat{\psi}_{I}\left(1_{\alpha}\right) \hat{H}_{\mathrm{ext}}\left(t_{1 \alpha}\right) \hat{\psi}_{I}^{\dagger}\left(2_{\beta}\right)\right] .
\end{aligned}
$$

In the second term of the right-hand side of Eq. (26) we have the time derivative of the electron annihilation operator that is, in the interaction picture,

$$
\begin{aligned}
i \partial_{t_{1}} \hat{\psi}_{I}(1) & =\left[\hat{\psi}_{I}(1), \hat{H}_{I}\left(t_{1}\right)\right] \\
& =\left[\hat{h}(1)-\frac{i}{c} \hat{\mathbf{A}}(1) \cdot \nabla_{1}+\hat{\phi}(1)\right] \hat{\psi}_{I}(1) .
\end{aligned}
$$

$\hat{H}_{I}$ is the representation, in the interaction picture, of $\hat{H}_{\text {ext }}(t)$, defined in Eq. (12), while the third and fourth terms in Eq. (26) give

$$
\begin{aligned}
i \alpha \mathrm{T}_{C}\left[\hat{S}_{C} \hat{H}_{\mathrm{ext}}\left(t_{1}\right) \hat{\psi}_{I}\left(1_{\alpha}\right) \hat{\psi}_{I}^{\dagger}\left(2_{\beta}\right)\right] & \\
& \quad-i \alpha \mathrm{T}_{C}\left[\hat{S}_{C} \hat{\psi}_{I}\left(1_{\alpha}\right) \hat{H}_{\mathrm{ext}}\left(t_{1}\right) \hat{\psi}_{I}^{\dagger}\left(2_{\beta}\right)\right] \\
= & -i \alpha \phi_{\mathrm{ext}}(1) \mathrm{T}_{c}\left[\hat{S}_{c} \hat{\psi}\left(1_{\alpha}\right) \hat{\psi}^{\dagger}\left(2_{\beta}\right)\right] .
\end{aligned}
$$

Equations (26)-(28), finally, lead to the following equation of motion for $G$ :

$$
\begin{aligned}
& {\left[i \partial_{t_{1}}-\hat{h}(1)-\frac{i}{c}\left\langle\hat{\mathbf{A}}\left(1_{\alpha}\right)\right\rangle_{C} \cdot \nabla_{1}-U\left(1_{\alpha}\right)\right] G\left(1_{\alpha}, 2_{\beta}\right)} \\
& \quad=\delta\left(1_{\alpha}-2_{\beta}\right)+\left.i \frac{\delta G\left(1_{\alpha}, 2_{\beta}\right)}{\delta \rho_{\mathrm{ext}}\left(3_{\gamma}\right)}\right|_{3_{\gamma}=1_{\alpha}}-\left.\nabla_{1} \cdot \frac{\delta G\left(1_{\alpha}, 2_{\beta}\right)}{\delta \mathbf{J}_{\mathrm{ext}}\left(3_{\gamma}\right)}\right|_{3_{\gamma}=1_{\alpha}} .
\end{aligned}
$$

In Eq. (29), we defined the total potential

$$
U(1)=\phi_{\mathrm{ext}}(1)+\langle\hat{\phi}(1)\rangle_{C},
$$

and used the following identities for the functional derivatives

$$
\begin{aligned}
\frac{\delta G\left(1_{\alpha}, 2_{\beta}\right)}{\delta \rho_{\mathrm{ext}}\left(3_{\gamma}\right)}= & i \gamma\left\langle\hat{\phi}_{\gamma}\left(3_{\gamma}\right)\right\rangle_{C} G\left(1_{\alpha}, 2_{\beta}\right) \\
& -\beta \gamma\left\langle\hat{\phi}\left(3_{\gamma}\right) \hat{\psi}\left(1_{\alpha}\right) \hat{\psi}^{\dagger}\left(2_{\beta}\right)\right\rangle_{C}
\end{aligned}
$$

and

$$
\begin{aligned}
\frac{\delta G\left(1_{\alpha}, 2_{\beta}\right)}{\delta \mathbf{J}_{\mathrm{ext}}\left(3_{\gamma}\right)}= & i \frac{\gamma}{c}\left\langle\hat{\mathbf{A}}\left(3_{\gamma}\right)\right\rangle_{C} G\left(1_{\alpha}, 2_{\beta}\right) \\
& +\frac{\beta \gamma}{c}\left\langle\hat{\mathbf{A}}\left(3_{\gamma}\right) \hat{\psi}\left(1_{\alpha}\right) \hat{\psi}^{\dagger}\left(2_{\beta}\right)\right\rangle_{C} .
\end{aligned}
$$

Equation (29) can be also used to define the noninteracting Green's function in order to rewrite it as Dyson equation or, alternatively, as BKE. Indeed, we start by noticing that $G_{0}$ is the solution of Eq. (29) when $\frac{\delta G}{\delta \rho_{\mathrm{ext}}}=\frac{\delta G}{\delta \mathbf{J}_{\mathrm{ext}}}=0$ :

$$
\left[i \partial_{t_{1}}-h_{\mathrm{ext}}\left(1_{\alpha}\right)\right] G_{0}\left(1_{\alpha}, 2_{\beta}\right)=\delta\left(1_{\alpha}-2_{\beta}\right)
$$

with

$$
h_{\mathrm{ext}}(1)=h(1)-\frac{i}{c}\langle\hat{\mathbf{A}}(1)\rangle_{C} \cdot \nabla_{1}-U(1) .
$$

Equations (33) and (34) imply that

$$
G_{0}^{-1}\left(1_{\alpha}, 2_{\beta}\right)=\left[i \partial_{t_{1}}-h_{\mathrm{ext}}\left(1_{\alpha}\right)\right] \delta\left(1_{\alpha}-2_{\beta}\right) .
$$

By using Eq. (35) we can further rewrite Eq. (29) in terms of the bare and of the fully interacting Green's functions. This is, clearly, a step towards the final form of the Dyson-type equation:

$$
\begin{aligned}
G\left(1_{\alpha}, 2_{\beta}\right)= & G_{0}\left(1_{\alpha}, 2_{\beta}\right)+G_{0}\left(1_{\alpha}, 4_{\sigma}\right) \\
& \times\left[\left.i \frac{\delta G\left(4_{\sigma}, 2_{\beta}\right)}{\delta \rho_{\mathrm{ext}}\left(3_{\gamma}\right)}\right|_{3_{\gamma}=4_{\sigma}}-\left.\nabla_{1} \cdot \frac{\delta G\left(4_{\sigma}, 2_{\beta}\right)}{\delta \mathbf{J}_{\mathrm{ext}}\left(3_{\gamma}\right)}\right|_{3_{\gamma}=4_{\sigma}}\right] .
\end{aligned}
$$

The electromagnetic field and the nuclei are now quantized, so the entire theoretical scheme is closed only when the equations of motion for their propagators are introduced. Indeed, it is interesting to note that all different effects induced by the mutual interactions (e-e, e-p, e- $\gamma$ ) will mix together in the dressing of the total fields connected to the external sources. This dressing will involve the ensemble of electrons, photons, and phonons via oscillations (collective and not) described by the corresponding response functions. Indeed, as it will be clear shortly, at difference with the well-known purely electronic case new response and vertex functions must be introduced.

\section{B. Equation of motion for the electromagnetic potentials}

The equation of motion for the fields is obtained by taking the macroscopic average of $U$ and $\langle\hat{\mathbf{A}}\rangle$. Indeed, from classical electrodynamics, we know that the scalar potential $U$ is the solution of the Poisson equation

$$
\nabla^{2} U(1)=-4 \pi\left[\rho_{\mathrm{ext}}(1)+\langle\hat{n}(1)\rangle_{C}\right],
$$

while the expectation value of the vector potential $\langle\hat{\mathbf{A}}\rangle$ satisfies the equation of motion

$$
\begin{aligned}
\left(\frac{1}{c^{2}} \partial_{t_{1}}^{2}-\nabla_{1}^{2}\right)\langle\hat{\mathbf{A}}(1)\rangle_{C} & =\frac{4 \pi}{c} \mathbf{J}_{\text {tot }}^{\perp}(1) \\
& =\frac{4 \pi}{c} \int d 2 \overleftrightarrow{\delta} \overleftrightarrow{\delta}_{(1,2) \mathbf{J}_{\text {tot }}(2)}
\end{aligned}
$$

The $\perp$ superscript means that only the transverse part of the current enters in the Eq. (38). This is obtained from the total current vector using the transverse delta function

$$
\begin{aligned}
\delta_{\alpha \beta}^{\perp}(1,2)= & \delta\left(t_{1}-t_{2}\right)\left[\delta\left(\mathbf{r}_{1}-\mathbf{r}_{2}\right) \delta_{\alpha \beta}\right. \\
& \left.+\frac{1}{4 \pi} \frac{\partial}{\partial r_{1, \alpha}}\left(\frac{1}{\left|\mathbf{r}_{1}-\mathbf{r}_{2}\right|} \frac{\partial}{\partial r_{2, \beta}}\right)\right] .
\end{aligned}
$$




\section{Response functions, vertex functions, and self-energies}

The equation of motion for $G$ [Eq. (29)] is still written in an obscure way and the different physical ingredients describing the complex many-body dynamics are hidden inside the functional derivatives $\left[\frac{\delta G\left(4_{\sigma}, 2_{\beta}\right)}{\delta \rho_{\mathrm{ext}(3)}}\right.$ and $\left.\frac{\delta G\left(4_{\sigma}, 2_{\beta}\right)}{\delta \mathbf{J}_{\mathrm{ext}}\left(3_{\gamma}\right)}\right]$. In this section, we investigate the structure of these derivatives by introducing the longitudinal and transverse response function and vertex functions.

We start by noticing that, as a consequence of the Green's function definition, it follows that

$$
\begin{aligned}
G\left(1_{\alpha}, 2_{\beta}\right) G^{-1}\left(2_{\beta}, 3_{\gamma}\right) & =\delta\left(1_{\alpha}-2_{\beta}\right) \Rightarrow \frac{\delta G\left(1_{\alpha}, 2_{\beta}\right)}{\delta h\left(3_{\gamma}\right)} \\
& =-G\left(1_{\alpha}, 4_{\eta}\right)\left[\frac{\delta G^{-1}\left(4_{\eta}, 5_{\xi}\right)}{\delta h\left(3_{\gamma}\right)}\right] G\left(5_{\xi}, 2_{\beta}\right)
\end{aligned}
$$

for any well-behaved function $h$. In Eq. (40) we have introduced the right $G^{-1}$ function that acts on the right arguments of $G$. Note that an alternative formulation can be introduced by using the left $G^{-1}$ defined in such a way that $G^{-1}\left(1_{\alpha}, 2_{\beta}\right) G\left(2_{\beta}, 3_{\gamma}\right)=\delta\left(1_{\alpha}-2_{\beta}\right)$ [53].

We can now apply a second rule that states that

$$
\frac{\delta G^{-1}\left(4_{\eta}, 5_{\xi}\right)}{\delta h\left(3_{\gamma}\right)}=\frac{\delta G^{-1}\left(4_{\eta}, 5_{\xi}\right)}{\delta g\left(6_{\sigma}\right)} \frac{\delta g\left(6_{\sigma}\right)}{\delta h\left(3_{\gamma}\right)} .
$$

From Eqs. (40) and (41), we get that, by using $h=\rho$ and $g=U$ [see Eq. (30)],

$$
\begin{aligned}
\left.\frac{\delta G\left(1_{\alpha}, 2_{\beta}\right)}{\delta \rho_{\mathrm{ext}}\left(3_{\gamma}\right)}\right|_{3_{\gamma}=1_{\alpha}}= & G\left(1_{\alpha}, 4_{\eta}\right) \gamma\left(4_{\eta}, 5_{\xi}, 6_{\sigma}\right) \\
& \times W\left(6_{\sigma}, 1_{\alpha}\right) G\left(5_{\xi}, 2_{\beta}\right) .
\end{aligned}
$$

With Eq. (42) we can now introduce the scalar (longitudinal) part of the total self-energy operator $\Sigma_{\text {long }}$, defined as

$$
\left.i \frac{\delta G\left(1_{\alpha}, 2_{\beta}\right)}{\delta \rho_{\mathrm{ext}}\left(3_{\gamma}\right)}\right|_{3_{\gamma}=1_{\alpha}}=\Sigma_{\text {long }}\left(1_{\alpha}, 3_{\gamma}\right) G\left(3_{\gamma}, 2_{\beta}\right),
$$

and, from Eq. (42), it follows that

$$
\Sigma_{\text {long }}\left(1_{\alpha}, 2_{\gamma}\right) \equiv G\left(1_{\alpha}, 3_{\gamma}\right) \gamma\left(3_{\gamma}, 2_{\beta}, 4_{\sigma}\right) W\left(4_{\sigma}, 1_{\alpha}\right) .
$$

The definition of the self-energy operator naturally introduces scalar response function

$$
W(1,2)=\frac{\delta U(1)}{\delta \rho_{\mathrm{ext}}(2)},
$$

and the longitudinal vertex function $\gamma(1,2,3)$,

$$
\gamma(1,2,3)=-\frac{\delta G^{-1}(1,2)}{\delta U(3)} .
$$

A similar procedure can be applied to the other derivative that appears in Eq. (29), $\frac{\delta G}{\delta \mathbf{J}_{\mathrm{ext}}}$. At difference with the charge derivative, this new term is a vector and we use Greek symbols $(\alpha, \beta, \ldots)$ to label its Cartesian components. By applying the following substitutions to Eqs. (40) and (41),

$$
\frac{\delta}{\delta \rho_{\mathrm{ext}}} \Rightarrow \frac{\delta}{\delta J_{\mathrm{ext}, \alpha}}
$$

$$
\frac{\delta}{\delta U} \Rightarrow \frac{\delta}{\delta A_{\alpha}}
$$

we get that

$$
\begin{aligned}
\frac{\delta G\left(1_{\alpha}, 2_{\beta}\right)}{\left.\delta J_{\mathrm{ext}, \lambda}\left(3_{\gamma}\right)\right|_{3_{\gamma}=1_{\alpha}}=} & -\sum_{\theta=1}^{3} G\left(1_{\alpha}, 4_{\eta}\right) \frac{\delta G^{-1}\left(4_{\eta}, 5_{\xi}\right)}{\delta\left\langle\hat{A}_{\theta}\left(6_{\sigma}\right)\right\rangle_{C}} \\
& \times \frac{\delta\left\langle\hat{A}_{\theta}\left(6_{\sigma}\right)\right\rangle_{C}}{\delta J_{\mathrm{ext}, \lambda}\left(1_{\alpha}\right)} G\left(5_{\xi}, 2_{\beta}\right) .
\end{aligned}
$$

Equation (49) allows to introduce the second important term in the total self-energy corresponding to its transverse contribution $\Sigma_{\text {trans }}$ :

$$
\begin{aligned}
\Sigma_{\text {trans }}\left(1_{\alpha}, 2_{\beta}\right)= & -\left.\sum_{\lambda=1}^{3} \nabla_{1, \lambda} \frac{\delta G\left(1_{\alpha}, 2_{\beta}\right)}{\delta J_{\text {ext }, \lambda}\left(3_{\gamma}\right)}\right|_{3_{\gamma}=1_{\alpha}} \\
= & i \sum_{\lambda, \theta=1}^{3} \Pi_{\lambda}(1,3) G\left(1_{\alpha}, 4_{\eta}\right) \Gamma_{\theta}\left(4_{\eta}, 5_{\xi}, 6_{\sigma}\right) \\
& \times\left.\mathcal{D}_{\theta \lambda}\left(6_{\sigma}, 3_{\beta}\right) G\left(5_{\xi}, 2_{\beta}\right)\right|_{3_{\gamma}=1_{\alpha}}
\end{aligned}
$$

where

$$
\Pi(1,2)=-\frac{i}{2}\left(\nabla_{1}-\nabla_{2}\right)=\Pi(1)+\Pi^{*}(2) .
$$

The transverse self-energy (50) further introduces two functions. The transverse photon propagator, which we will call $\overleftrightarrow{\mathcal{D}}(1,2)$

$$
\overleftrightarrow{\mathcal{D}}(1,2)=-\frac{c}{4 \pi} \frac{\delta\langle\hat{\mathbf{A}}(1)\rangle_{C}}{\delta \mathbf{J}_{\text {ext }}(2)}
$$

and the transverse vertex (vectorial) function $\Gamma$,

$$
\Gamma(1,2,3)=-\frac{4 \pi}{c} \frac{\delta G^{-1}(1,2)}{\delta\langle\hat{\mathbf{A}}(3)\rangle} .
$$

We notice now that the longitudinal screened potential and vertex are connected, via Eq. (37), to the total density. This includes both an electronic and a nuclear component. It is then clear that the nuclear motion will enter directly in the electronic dynamics via the longitudinal components.

This sharp separation between longitudinal and transverse components of the theory is possible thanks to the use of the Coulomb gauge. This allows the electromagnetic field to be separated into two independent parts and the entire theory follows the same structure. As we will be seeing shortly, the final expression for the self-energy will also be split in a longitudinal and in a transverse part (see Fig. 3).

The final step of this section is obtained by using Eqs. (50) and (46) to define the total self-energy $\Sigma=\Sigma_{\text {long }}+\Sigma_{\text {trans }}$. This can be introduced in Eqs. (36) and (29) to obtain the final form of the equation of motions for the $G$ written as time derivative

$$
\begin{aligned}
& {\left[i \partial_{t_{1}}-h_{\mathrm{ext}}(1)\right] G\left(1_{\alpha}, 2_{\beta}\right)} \\
& \quad=\delta\left(1_{\alpha}-2_{\beta}\right)+\Sigma\left(1_{\alpha}, 3_{\gamma}\right) G\left(3_{\gamma}, 2_{\beta}\right),
\end{aligned}
$$

and as Dyson-type equation

$$
G\left(1_{\alpha}, 2_{\beta}\right)=G_{0}\left(1_{\alpha}, 2_{\beta}\right)+G_{0}\left(1_{\alpha}, 3_{\gamma}\right) \Sigma\left(3_{\gamma}, 4_{\eta}\right) G\left(4_{\eta}, 2_{\beta}\right) .
$$




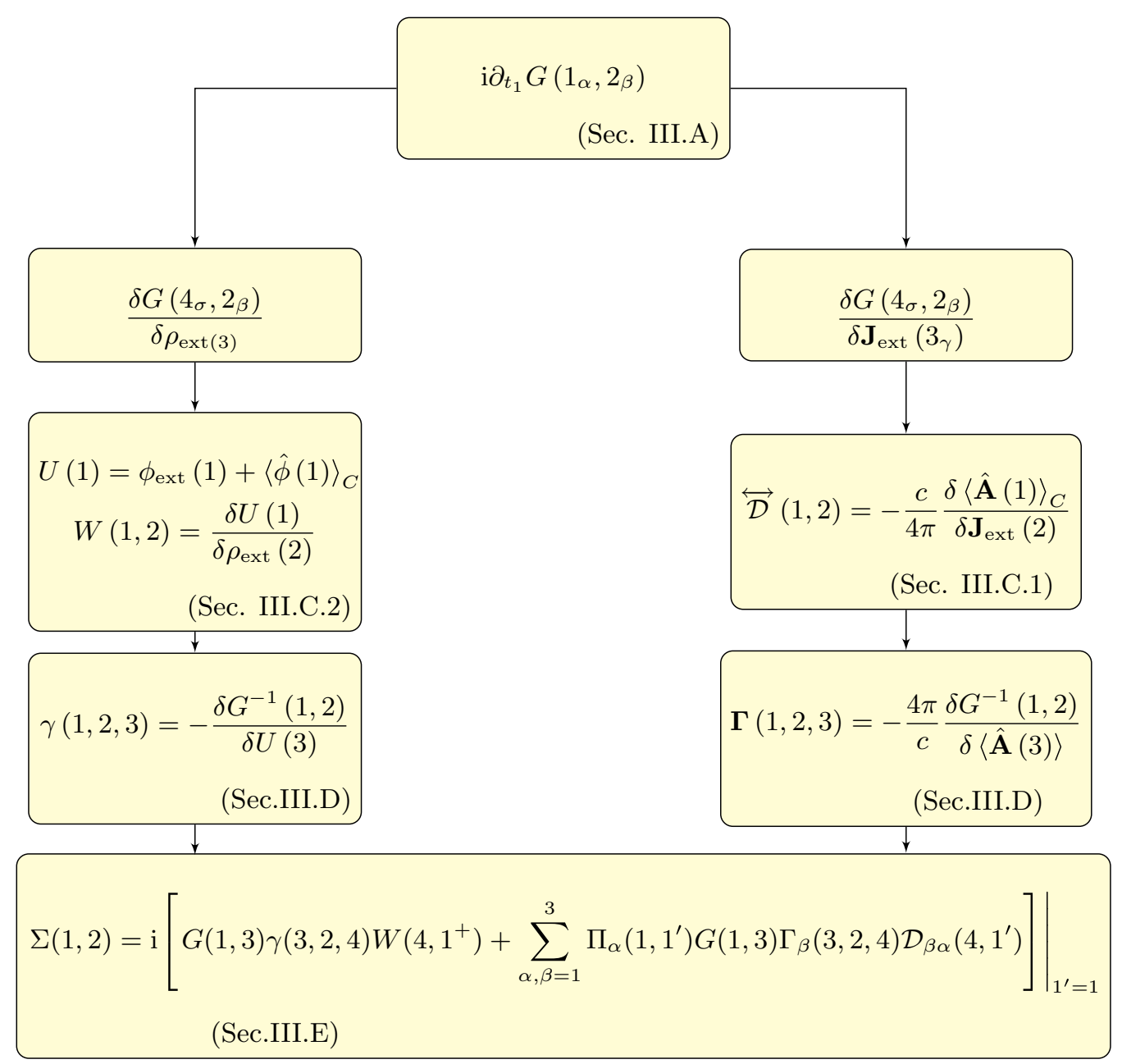

FIG. 3. Schematic representation of the mathematical procedure followed to get the Dyson equation within the framework of the Hedin's equations. The initial derivative is split in longitudinal (left) and transverse (right) contributions.

Equations (54) and (55) are equivalent and represent two different formulations of the well-known KBE.

From the equation of motion for the scalar and for the vector potential, we can now derive the equations governing the response functions. Indeed, these functions describe the change in the observables due to the total (external plus induced) perturbations and provide a connection between Eqs. (37), (38), and (29). We analyze, now, separately the two contributions: transverse (photon induced) and longitudinal (electronic and phonon mediated).

\section{Photon-induced response function}

The photon-induced response function represents the transverse photon propagator. We start from Eq. (38) and divide the total current in an external and an induced part: $\mathbf{J}_{\text {tot }}=$ $\mathbf{J}_{\text {ext }}+\mathbf{J}_{\text {ind }}$. Thus, we can express the solution of Eq. (38) as

$$
\langle\hat{\mathbf{A}}(1)\rangle_{C}=-\frac{4 \pi}{c} \int d 2 \overleftrightarrow{\mathcal{D}}_{0}(1,2)\left[\mathbf{J}_{\mathrm{ext}}(2)+\mathbf{J}_{\text {ind }}(2)\right]
$$

where $\mathcal{D}_{0}(1,2)$ is the free transverse photon propagator solution of

$$
\left(\frac{1}{c^{2}} \partial_{t_{1}}^{2}-\nabla_{1}^{2}\right) \overleftrightarrow{\mathcal{D}}_{0}(1,2)=\overleftrightarrow{\delta} \perp(1,2)
$$

By applying the chain rule (40), we derive the equation of motion for the transverse photon propagator

$$
\overleftrightarrow{\mathcal{D}}(1,2)=\overleftrightarrow{\mathcal{D}}_{0}(1,2)+\overleftrightarrow{\mathcal{D}}_{0}(1,3) \overleftrightarrow{P}(3,4) \overleftrightarrow{\mathcal{D}}(4,2)
$$

where we have defined the transverse photon polarization $\overleftrightarrow{P}(3,4)$

$$
\overleftrightarrow{P}(1,2)=-\frac{4 \pi}{c} \frac{\delta \mathbf{J}_{\text {ind }}(1)}{\delta\langle\hat{\mathbf{A}}(2)\rangle_{C}} .
$$

To obtain the equation for the transverse polarization we need a microscopic expression for the induced current $\mathbf{J}_{\text {ind }}$. This is related to the electronic Green's function by

$$
\begin{aligned}
\mathbf{J}_{\text {ind }}(1) & =\langle\hat{\mathbf{J}}(1)\rangle_{C}-\langle\hat{\rho}(1) \hat{\mathbf{A}}(1)\rangle_{C} \\
& =\left.\mathrm{i} \Pi\left(1,1^{\prime}\right) G\left(1,1^{\prime}\right)\right|_{1^{\prime}=1^{+}} .
\end{aligned}
$$

In deriving Eq. (60), we have omitted the $\rho \mathbf{A}$ term as we are considering averages on states with a fixed population of photons such that $\left\langle d_{\mathbf{q}+\mathbf{G}, \lambda}^{\dagger}\right\rangle_{C}=\left\langle d_{\mathbf{q}+\mathbf{G}, \lambda}\right\rangle_{C}=0$. This implies that $\langle\hat{\rho}(1) \hat{\mathbf{A}}(1)\rangle_{C}=0$. From Eqs. (59) and (52), we finally get a closed expression for the transverse photon polarization:

$$
P_{\alpha \beta}(1,2)=-\left.\mathrm{i} \Pi_{\alpha}\left(1,1^{\prime}\right) G(1,3) \Gamma_{\beta}(3,4,2) G\left(4,1^{\prime}\right)\right|_{1^{\prime}=1} .
$$




\section{Phonon-induced response function}

We now look into the screened Coulomb interaction $W$ defined in Eq. (45). By using Eq. (30), we obtain

$$
\begin{aligned}
W(1,2) & =w_{0}(1,2)+w_{0}(1,2)\left[\frac{\delta\langle\hat{\rho}(3)\rangle_{C}}{\delta \rho_{\mathrm{ext}}(2)}-\frac{\delta\langle\hat{N}(3)\rangle_{C}}{\delta \rho_{\mathrm{ext}}(2)}\right] \\
& =w_{0}(1,2)+w_{0}(1,3)\left[p_{\mathrm{e}}(3,4) W(4,2)-\frac{\delta\langle\hat{N}(3)\rangle_{C}}{\delta \rho_{\mathrm{ext}}(2)}\right],
\end{aligned}
$$

where $w_{0}(1,2)=\delta\left(t_{1}-t_{2}\right) v\left(\left|\mathbf{r}_{1}-\mathbf{r}_{2}\right|\right)$ is the bare Coulomb interaction, and $p_{\mathrm{e}}$ is the longitudinal electronic polarization:

$$
p_{\mathrm{e}}(1,2)=\frac{\delta\langle\hat{\rho}(1)\rangle_{C}}{\delta U(2)} .
$$

Equation (62) includes two contributions. One is coming from the electronic density (via $p_{e}$ ) and the other from the nuclear density (via $\delta N / \delta \rho$ ). Thus, by following Ref. [27], we separate $W$ into an electronic plus a nuclear part.

The electronic polarization is still defined, as in the purely electronic case [53], in terms of the electronic component of the total density

$$
\langle\hat{\rho}(1)\rangle_{C}=-i G\left(1,1^{+}\right),
$$

from which it immediately follows that

$$
p_{\mathrm{e}}(1,2)=i G(1,2) \gamma(3,4,2) G(4,1) .
$$

Again, in the derivation of Eq. (65) we used Eq. (40).

The new contribution in Eq. (62) is due to the change in the nuclear density induced by a change of the purely electronic part of the external charge. Indeed, this is the source of e-p interaction that describes the link between the nuclear motion and the electronic dynamics:

$$
\frac{\delta\langle\hat{N}(3)\rangle_{C}}{\delta \rho_{\text {ext }}(2)} .
$$

In order to link this quantity to the microscopic correlation functions, we need to introduce the nuclear density-density correlation function $D$ given by

$$
D(1,2)=-i\langle\Delta \hat{N}(1) \Delta \hat{N}(2)\rangle_{C},
$$

where the fluctuation of an operator is expressed as $\Delta \hat{O}=$ $\hat{O}-\langle\hat{O}\rangle$.

It is now possible to define an equation for $D$ thanks to the introduction, in Eq. (19), of the external nuclear charge $N_{\text {ext }}$. Indeed, we start by noticing that, as the interaction with the electronic density is $\int d^{3} r \hat{\phi}(\mathbf{r}, t) \rho_{\text {ext }}(\mathbf{r}, t)$, it follows that

$$
\frac{\delta\langle\hat{N}(1)\rangle_{C}}{\delta \rho_{\text {ext }}(2)}=-i\langle\Delta \hat{N}(1) \Delta \hat{\phi}(2)\rangle_{C} \text {. }
$$

If now we use the solution of the Poisson equation to rewrite $\Delta \hat{\phi}$ in terms of charge variations, we get

$$
\begin{aligned}
\frac{\delta\langle\hat{N}(1)\rangle_{C}}{\delta \rho_{\mathrm{ext}}(2)}= & i w_{0}(2,3)\left[\langle\Delta \hat{N}(1) \Delta \hat{N}(3)\rangle_{C}\right. \\
& \left.-\langle\Delta \hat{N}(1) \Delta \hat{\rho}(3)\rangle_{C}\right] .
\end{aligned}
$$

Using the same procedure as above we can evaluate a similar derivative $\frac{\delta\langle\hat{n}(1)\rangle}{\delta N_{\text {ext }}(2)}$. In this case, the interaction with $N_{\text {ext }}$ is $\int d^{3} r \hat{V}_{\mathrm{n}}(\mathbf{r}) N_{\mathrm{ext}}(\mathbf{r}, t)$. It follows, then, that

$$
\frac{\delta\langle\hat{n}(1)\rangle_{C}}{\delta N_{\text {ext }}(2)}=-i\left\langle\Delta \hat{n}(1) \Delta \hat{V}_{\mathrm{n}}(2)\right\rangle_{C} .
$$

By using again the solution of the Poisson equation we get that

$$
\frac{\delta\langle\hat{N}(3)\rangle}{\delta \rho_{\mathrm{ext}}(2)}=-\frac{\delta\langle\hat{n}(3)\rangle}{\delta N_{\mathrm{ext}}(2)} .
$$

We can, now, easily evaluate Eq. (66) via Eq. (71). Taking into account the definition of $\hat{n}$ we can use the functional derivative chain rule to obtain a Dyson-type equation

$$
\begin{aligned}
w_{0}(1,3) \frac{\delta\langle\hat{n}(3)\rangle}{\delta N_{\mathrm{ext}}(2)} \\
=w_{0}(1,3) D(3,4) w_{0}(4,2)+w_{0}(1,3) \frac{\delta\langle\hat{\rho}(3)\rangle}{\delta N_{\mathrm{ext}}(2)} \\
=w_{0}(1,3) D(3,4) w_{0}(4,2) \\
\quad+w_{0}(1,3) \frac{\delta\langle\hat{\rho}(3)\rangle}{\delta U(4)} \frac{\delta U(4)}{\delta\langle\hat{n}(5)\rangle} \frac{\delta\langle\hat{n}(5)\rangle}{\delta N_{\mathrm{ext}}(2)} \\
=w_{0}(1,3) D(3,4) w_{0}(4,2) \\
\quad+w_{0}(1,3) p_{\mathrm{e}}(3,4) w_{0}(4,5) \frac{\delta\langle\hat{n}(5)\rangle}{\delta N_{\mathrm{ext}}(2)} .
\end{aligned}
$$

Iterating the equation leads to the following solution:

$$
\begin{aligned}
& w_{0}(1,3) \frac{\delta\langle\hat{n}(3)\rangle}{\delta N_{\mathrm{ext}}(2)} \\
& \quad=\left[1-w_{0} p_{\mathrm{e}}\right]^{-1}(1,3) w_{0}(3,4) D(4,5) w_{0}(5,2),
\end{aligned}
$$

which we can then replace in Eq. (62) obtaining the final expression for the total screened interaction

$$
\begin{aligned}
W(1,2)= & w_{0}(1,2)+w_{0}(1,3) p_{\mathrm{e}}(3,4) W(4,2) \\
& +\left[1-w_{0} p_{\mathrm{e}}\right]^{-1}(1,3) w_{0}(3,4) D(4,5) w_{0}(5,2),
\end{aligned}
$$

and solving it in order to $W$, which leads to two separate contributions

$$
W(1,2)=W_{\mathrm{e}}(1,2)+W_{\mathrm{ph}}(1,2) .
$$

The first term $W_{\mathrm{e}}$ accounts for the interactions between the electrons and has no direct contribution from the nuclei

$$
W_{\mathrm{e}}(1,2)=\left[1-w_{0} p_{\mathrm{e}}\right]^{-1}(1,3) w_{0}(3,2),
$$

while the second term introduces the effects of the contribution from the nuclear density fluctuations

$$
W_{\mathrm{ph}}(1,2)=\left[1-w_{0} p_{\mathrm{e}}\right]^{-1}(1,3) W_{\mathrm{e}}(3,4) D(4,5) w_{0}(5,2) .
$$

\section{Vertex functions}

In Eqs. (42) and (49), we have introduced a longitudinal and a transverse vertex function. The equations of motion that govern their dynamics can be easily found by differentiating the inverse of the Dyson equation (36):

$$
G^{-1}(1,2)=G_{0}^{-1}(1,2)-\Sigma(1,2) .
$$


Thus, we can write for the longitudinal vertex, which is given by Eq. (46), the following equation:

$$
\begin{aligned}
\gamma(1,2,3)= & -\frac{\delta G^{-1}(1,2)}{\delta U(3)}=\delta(1,2) \delta(1,3) \\
& +\frac{\delta \Sigma(1,2)}{\delta G(4,5)} G(4,6) \gamma(6,7,3) G(7,5),
\end{aligned}
$$

and for the transverse part [Eq. (52)] we have that

$$
\begin{aligned}
\Gamma(1,2,3)= & \Pi(1) \delta(1,3) \delta(1,2) \\
& +\frac{\delta \Sigma(1,2)}{\delta G(4,5)} G(4,6) \Gamma(6,7,3) G(7,5) .
\end{aligned}
$$

Vertex and response functions are the ingredients we need to close the equation of motion for the Green's function in a set of integrodifferential equations. At this stage, it is important to note that through the functional derivative $\frac{\delta \Sigma}{\delta G}$ all interactions mix together in a complex dynamics.

\section{Final form of the Hedin's equations}

We are now ready to present the full set of the Hedin's equations. Those are schematically shown in Fig. 5. The first equation gives us the electron propagator $G$ :

$$
G(1,2)=G_{0}(1,2)+G_{0}(1,3) \Sigma(3,4) G(4,2) .
$$

The second equation allows us to obtain the self-energy

$$
\begin{aligned}
\Sigma(1,2)= & \mathrm{i}\left[G(1,3) \gamma(3,2,4) W\left(4,1^{+}\right)\right. \\
& \left.+\sum_{\alpha, \beta=1}^{3} \Pi_{\alpha}\left(1,1^{\prime}\right) G(1,3) \Gamma_{\beta}(3,2,4) \mathcal{D}_{\beta \alpha}\left(4,1^{\prime}\right)\right]\left.\right|_{1^{\prime}=1},
\end{aligned}
$$

in which a new term on the right-hand side is present, due to the fact that the electromagnetic field is now quantized. The fourth and fifth equations describe the screened Coulomb interaction

$$
W(1,2)=W_{\mathrm{e}}(1,2)+W_{\mathrm{ph}}(1,2),
$$

and the transverse photon propagator

$$
\overleftrightarrow{\mathcal{D}}(1,2)=\overleftrightarrow{\mathcal{D}}_{0}(1,2)+\overleftrightarrow{\mathcal{D}}_{0}(1,3) \overleftrightarrow{P}(3,4) \overleftrightarrow{\mathcal{D}}(4,2)
$$

In order to evaluate them, we need the longitudinal and transverse polarizations

$$
p_{\mathrm{e}}(1,2)=i G(1,2) \gamma(3,4,2) G(4,1)
$$

and

$$
P_{\alpha \beta}(1,2)=\left.\mathrm{i} \Pi_{\alpha}\left(1,1^{\prime}\right) G(1,3) \Gamma_{\beta}(3,4,2) G\left(4,1^{\prime}\right)\right|_{1^{\prime}=1} .
$$

These equations form the sixth and seventh Hedin's equations that introduce the corresponding vertex functions, whose equations of motion represent the last two Hedin equations

$$
\gamma(1,2,3)=\delta(1,2) \delta(1,3)+\frac{\delta \Sigma(1,2)}{\delta G(4,5)} G(4,6) \gamma(6,7,3) G(7,5)
$$
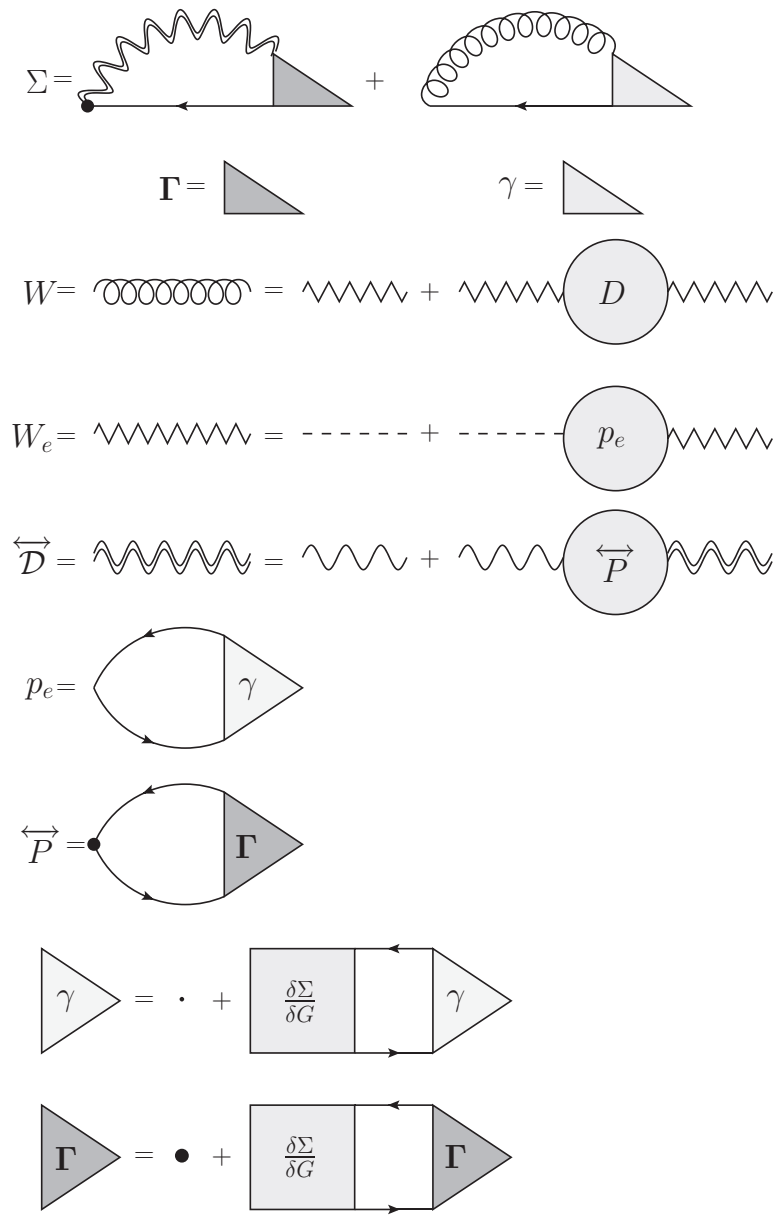

FIG. 4. Diagrammatic representation of the Hedin's equations. The bullet represents a $\Pi$ differential operator, defined in Eq. (51).

and

$$
\begin{aligned}
\Gamma(1,2,3)= & \Pi(1) \delta(1,3) \delta(1,2) \\
& +\frac{\delta \Sigma(1,2)}{\delta G(4,5)} G(4,6) \Gamma(6,7,3) G(7,5) .
\end{aligned}
$$

The only missing term is the equation of motion for $D$, the nuclear density-density correlation function. However, it is well known [27] that the electronic and nuclear parts of Hedin's equations can be safely decoupled. As a consequence, the $D$ propagator can be thought to be given and the purely electronic and photonic components of Hedin's equations can be solved self-consistently.

We have represented the new set of Hedin's equations in diagrammatic form in Fig. 4 and in schematic form in Fig. 5. This last representation also provides a method to solve the equations self-consistently. Starting from a reasonable approximation for the longitudinal and transverse parts of the self-energy $\Sigma$, we can then evaluate the longitudinal and the transverse vertex, respectively, $\gamma$ and $\boldsymbol{\Gamma}$. Following that, we can obtain the longitudinal and transverse polarizations $p_{\mathrm{e}}$ and $\overleftrightarrow{P}$ and incorporate the results in the photon propagator $\overleftrightarrow{\mathcal{D}}$ and, assuming that we have a good description for the nuclear oscillations in the form of the phonon propagator $D$, the screened longitudinal interaction $W$. As the last 


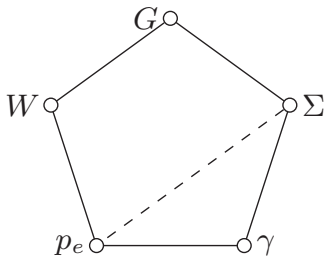

(a)

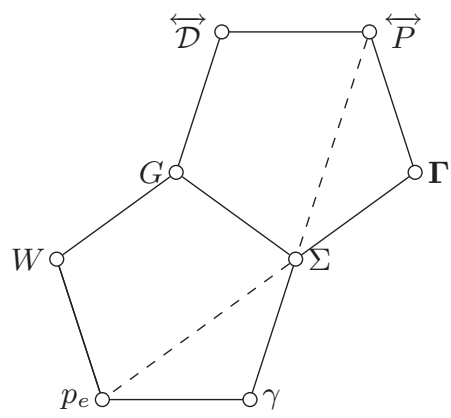

(b)

FIG. 5. Schematic representation of the different formulations of Hedin's equations. (a) Longitudinal case. This is well known and widely used in the literature in its equilibrium and out-of-equilibrium versions. When the interaction with the quantized electromagnetic field is switched on, a new pentagon must be added [case (b)] where the corners are the photon propagator and the transverse electronic polarization and vertex function. The dashed lines correspond to the generalized $G W$ approximation where all vertex functions are neglected.

step of the cycle, we can obtain the expression for the Green's function $G$. This is analogous to the usual description for a self-consistent MBPT calculation, only that in this case we have two subcycles to evaluate: one for the transverse and another for the longitudinal electromagnetic field.

\section{THE $G W$ APPROXIMATION}

The $G W$ approximation [25] is based on the assumption that the corrections to the vertex can be ignored. In the present case, it corresponds to ignore the second term in Eqs. (87) and (88) so that the vertex functions acquire a simple form

$$
\Gamma^{G W}(1,2,3)=\Pi(1) \delta(1,3) \delta(1,2)
$$

and

$$
\gamma^{G W}(1,2,3)=\delta(1,2) \delta(1,3) .
$$

As a consequence, both the longitudinal and transverse polarization functions turn into an independent-particle representation

$$
p_{\mathrm{e}}^{G W}(1,2)=i G(1,2) G(2,1)
$$

and

$$
P_{\alpha \beta}^{G W}(1,2)=\left.i \Pi_{\alpha}\left(1,1^{\prime}\right) G(1,2) \Pi_{\beta}(2) G\left(2,1^{\prime}\right)\right|_{1^{\prime}=1} .
$$
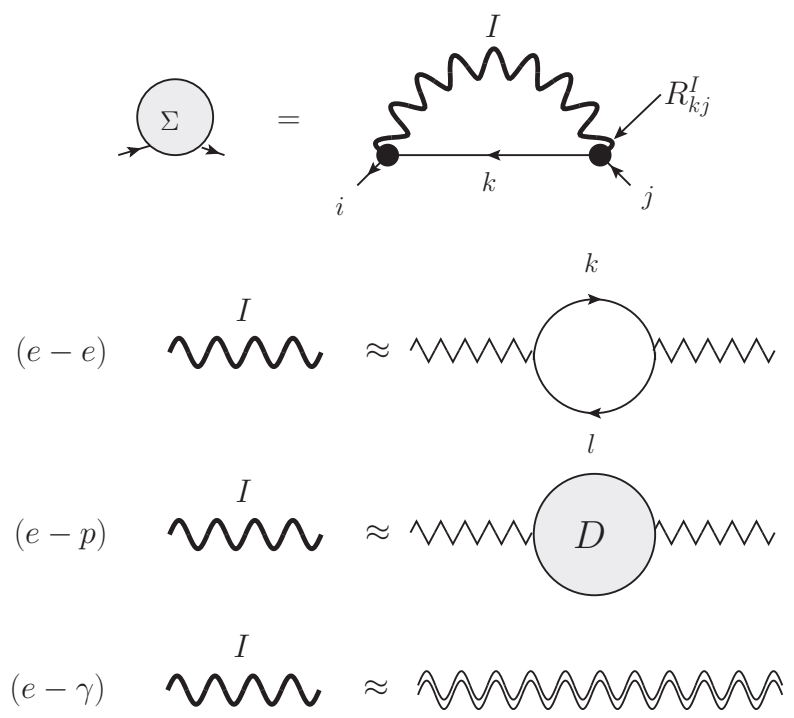

FIG. 6. The generalized $G W$ approximation for the three kinds of interactions: e-e, e-p, and e- $\gamma$. The wiggled propagator in the e-e case represents a statically screened interaction, as explained in Ref. [38].

The final expression for the electronic self-energy in the $G W$ approximation is then given by

$$
\begin{aligned}
\Sigma^{G W}(1,2)=i & {\left[G(1,2) W(2,1)+\sum_{\alpha, \beta=1}^{3} \Pi_{\alpha}\left(1,1^{\prime}\right)\right.} \\
& \left.\times G(1,2) \Pi_{\beta}(2) \mathcal{D}_{\beta \alpha}\left(2,1^{\prime}\right)\right]\left.\right|_{1^{\prime}=1} .
\end{aligned}
$$

The longitudinal $G W$ self-energy has been extensively studied and its formulation for the e-e and e-p parts is well known (see Fig. 6).

\section{A SIMPLIFIED FORMULATION USING A SINGLE-TIME DENSITY MATRIX REPRESENTATION}

The BKE [Eq. (54)] is very hard to solve for practical applications and in realistic materials. The reason is the complex two-times dependence and spatial nonlocality that enormously increases the complexity of the problem compared to the more common methods used in the $a i$-MBPT scheme [13].

An approach that is attracting great interest is based on the reduction of the complex equation for $G\left(1_{\alpha}, 2_{\beta}\right)$ in a closed equation for the density matrix $\rho(1) \equiv-i G\left(1_{-}, 1_{+}\right)=$ $-i G^{<}(1,2)$. This approach is based on the GBKA and, in the most recent approaches, on the CCA.

The aim of this section is to extend the derivation of the reduced $\mathrm{KBE}$ to the general case of the simultaneous presence of e-e, e-p, and e- $\gamma$ interactions. We start by expressing all quantities in the second quantization basis

$$
\begin{aligned}
G(1,2) & =\sum_{i, j} \phi_{i}\left(\mathbf{r}_{1}\right) \phi_{j}^{*}\left(\mathbf{r}_{2}\right) G_{i j}\left(t_{1}, t_{2}\right), \\
\Sigma(1,2) & =\sum_{i, j} \phi_{i}\left(\mathbf{r}_{1}\right) \phi_{j}^{*}\left(\mathbf{r}_{2}\right) \Sigma_{i j}\left(t_{1}, t_{2}\right),
\end{aligned}
$$


in such a way to remove the spatial dependence and concentrate our attention on the time arguments. We will discuss in Sec. VIII the properties of the single-particle basis used in (95).

In this single-particle basis, the $\mathrm{KBE}$ for $G^{<}\left(t, t^{\prime}\right)$ can be rewritten in a compact, matrixlike form

$$
\begin{aligned}
{\left[i \partial_{t}-h_{\mathrm{ext}}(t)\right] G^{<}\left(t, t^{\prime}\right)=} & \int d \bar{t}\left[\Sigma^{r}(t, \bar{t}) G^{<}\left(\bar{t}, t^{\prime}\right)\right. \\
& \left.+\Sigma^{<}(t, \bar{t}) G^{a}\left(\bar{t}, t^{\prime}\right)\right]
\end{aligned}
$$

and its adjoint

$$
\begin{aligned}
& -i \partial_{t^{\prime}} G^{<}\left(t, t^{\prime}\right)-G^{<}\left(t, t^{\prime}\right) h_{\mathrm{ext}}\left(t^{\prime}\right) \\
& \quad=\int d \bar{t}\left[G^{r}(t, \bar{t}) \Sigma^{<}\left(\bar{t}, t^{\prime}\right)+G^{<}(t, \bar{t}) \Sigma^{a}\left(\bar{t}, t^{\prime}\right)\right] .
\end{aligned}
$$

In Eqs. (96) and (97), the retarded/advanced functions carry a superscript $r / a$ and are defined in terms of the lesser and greater functions according to

$$
X^{r}\left(t, t^{\prime}\right)=\left[X^{a}\left(t^{\prime}, t\right)\right]^{\dagger}=\theta\left(t-t^{\prime}\right)\left[X^{>}\left(t, t^{\prime}\right)-X^{<}\left(t, t^{\prime}\right)\right],
$$

where $X$ can be $G, \Sigma$, or any other two-time correlation function.

By subtracting the Eqs. (96) and (97), and taking the derivative on the macroscopic time axis $T=\left(t+t^{\prime}\right) / 2$ we get the equation of motion for $\rho(t)=-i G^{<}(T, T)$ :

$$
\frac{d}{d T} \rho(T)+i\left[h_{\mathrm{ext}}(T), \rho(T)\right]=-S[\{G\},\{\Sigma\}](T),
$$

where all the self-energy effects are embodied in the complex collision integral $S[\{G\},\{\Sigma\}](t)$ that is still a function of the $\lessgtr$ two-times Green's functions and self-energies:

$$
\begin{aligned}
S[\{G\},\{\Sigma\}](T)= & \int_{-\infty}^{T} d t\left[\Sigma^{>}(T, \bar{t}) G^{<}(\bar{t}, T)\right. \\
& \left.+G^{<}(T, \bar{t}) \Sigma^{>}(\bar{t}, T)-(\lessgtr \rightleftarrows \gtrless)\right] .
\end{aligned}
$$

In Eq. (100), the ( $\lessgtr \rightleftarrows \gtrless)$ indicates that the second part of the integral is obtained from the first part by exchanging $>(<)$ with $<(>)$.

The collision integral includes contributions from the local and nonlocal self-energies $\Sigma\left(t, t^{\prime}\right)=\Sigma_{s}(t)+\Sigma_{c}\left(t, t^{\prime}\right)$. The local part of the self-energy defines a coherent part of $S$ moving out of the time integral: $S[\{G\},\{\Sigma\}](T)=S^{\mathrm{coh}}(T)+$ $S^{\text {dyn }}[\{G\},\{\Sigma\}](T)$. Such splitting has already been discussed in Refs. [14,38].

The coherent part is embodied in the $h_{\text {ext }}(t)$ term which turns into

$$
\begin{aligned}
& i\left[h_{\mathrm{ext}}(T), \rho(T)\right]+S[\{G\},\{\Sigma\}](T) \\
& \quad=i[h(T), \rho(T)]+S^{\mathrm{coh}}[\rho](T)+S^{\mathrm{dyn}}[\{G\},\{\Sigma\}](T),
\end{aligned}
$$

with

$$
S^{\mathrm{coh}}[\rho](T)=\mathrm{i}\left[\Sigma_{S}(t), \rho(T)\right] .
$$

The different possible approximations to $\Sigma_{s}$ reflect the different kind of physics introduced in the dynamics and can already account for important effects. Different cases can be considered: (i) A mean-field potential that mimics the correlation effects. An example is DFT, where $\Sigma_{s}(t)$ is local in space and given by the sum of the Hartree and exchange-correlation potentials.

(ii) HF self-energy. In this case no correlation is included. The Hartree-Fock (HF) self-energy reads as

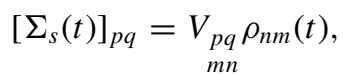

with the four-index tensor $V_{i j}=2\left[w_{0}\right]_{i m n j}-\left[w_{0}\right]_{i m j n}$ and $\left[w_{0}\right]_{i m n j}$ the two-electron bare Coulomb integrals:

$$
\left[w_{0}\right]_{i m n j} \equiv \int d \mathbf{r} d \mathbf{r}^{\prime} \phi_{i}^{*}(\mathbf{r}) \phi_{m}^{*}\left(\mathbf{r}^{\prime}\right) v\left(\mathbf{r}-\mathbf{r}^{\prime}\right) \phi_{n}\left(\mathbf{r}^{\prime}\right) \phi_{j}(\mathbf{r}) .
$$

(iii) Hartree plus a Coulomb hole and screened exchange (COHSEX) self-energy. In this case, correlation is included using a linear response approximation but dynamical effects are neglected. The COHSEX self-energy reads as

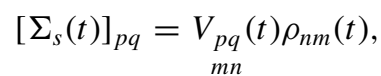

with now $V(t)_{i j}=2\left[w_{0}\right]_{i m n j}-W[\rho(t)]_{i m j n}$ and $W$ the screened Coulomb hole potential. This is routinely calculated in the random-phase approximation (RPA) where

$$
W[\rho]\left(\mathbf{r}, \mathbf{r}^{\prime}\right) \equiv \int d \overline{\mathbf{r}} \varepsilon_{\mathrm{RPA}}^{-1}[\rho](\mathbf{r}, \overline{\mathbf{r}}) v\left(\overline{\mathbf{r}}-\mathbf{r}^{\prime}\right) .
$$

The choice of the local part of the self-energy is essential as it already describes a large part of the level of correlation embodied in the many-body dynamics. It has been formally proved, for example, that the COHSEX self-energy describes in the linear regime the excitonic effects and reduces Eq. (99) to the well-known Bethe-Salpeter equation [14].

The collision integral $S^{\text {dyn }}(t)$ is nonlocal in time and its functional form is uniquely determined once an approximation for the correlation self-energy $\Sigma_{c}$ is made. Let us consider here the $G W$ approximation [Eq. (93)] that can be rewritten, by using Eq. (94), in a very general and compact form as

$$
\Sigma_{i j}^{\lessgtr}\left(t, t^{\prime}\right)=i \sum_{I}\left[\left(R^{I}\right)^{\dagger} G^{\lessgtr}\left(t, t^{\prime}\right) R^{I}\right]_{i j} W_{I}^{\lessgtr}\left(t, t^{\prime}\right) .
$$

In Eq. (107), $R^{I}$ and $G$ are matrices [see Eqs. (94) and (95)] and the product in the square brackets represents a matrix multiplication. In Eq. (107), and from this point onward, we use the Einstein convention that repeated indices are summed over. $W_{I}$, instead, is a scalar function. $R_{I}$ and $W$ have different definitions depending on the kind of interaction they are describing.

(a) In the e-p case $I=(\mathbf{q} \lambda)$ and represents the phonon branch $(\lambda)$ and momentum $(\mathbf{q})$ pair. It follows that

$$
R_{i j}^{I}=R_{i j}^{(\mathbf{q} \lambda)} \equiv \int d \mathbf{r} \phi_{i}^{*}(\mathbf{r}) \partial_{\mathbf{q} \lambda} V_{n}(\mathbf{r}) \phi_{j}(\mathbf{r}),
$$

with $\partial_{\mathbf{q} \lambda}$ the derivative of the bare ionic potential $\left(V_{n}\right)$ along the phonon state $(\mathbf{q} \lambda)$. Note that, if the present formalism is applied on top of DFT, $V_{n}(\mathbf{r})$ is replaced by the dressed self-consistent derivative of the DFT ionic potential as discussed in Ref. [28]. 
(b) In the e- $\gamma$ case the $I$ index represents the photon polarization index $(\lambda)$ and momentum $(\mathbf{q})$ and

$$
R_{i j}^{I}=\int d \mathbf{r} e^{i \mathbf{q}_{I} \cdot \mathbf{r}} \phi_{i}^{*}(\mathbf{r})\left(\mathbf{e}_{\lambda} \cdot \nabla\right) \phi_{j}(\mathbf{r}) .
$$

(c) In the e-e case the derivation of the analytic expression for $R^{I}$ is more mathematically involved. At the same time, however, this procedure has been extensively studied in the literature. We take as reference $[38,56]$ to rewrite the out-ofequilibrium screened e-e interaction as an effective interaction with a time-dependent plasma of electron-hole pairs with scattering amplitudes given by

$$
R_{i j}^{I}=\int d \mathbf{r} d \mathbf{r}^{\prime} \phi_{i}^{*}(\mathbf{r}) W[\rho]\left(\mathbf{r}, \mathbf{r}^{\prime}\right) \phi_{j}(\mathbf{r})\left[\Phi_{I}\left(\mathbf{r}^{\prime}\right)\right],
$$

where $I=(k, l)$ and $\Phi_{I}(\mathbf{r}) \equiv \phi_{k}^{*}(\mathbf{r}) \phi_{l}(\mathbf{r})$. In this case, indeed, the elemental excitations are electron-hole pairs $(k, l)$.

In all of the three cases above, $W$ has the form

$$
W_{I}^{\lessgtr}\left(t, t^{\prime}\right) \equiv W_{I}^{\lessgtr}\left(t-t^{\prime}\right) \equiv(-i) N_{I}^{ \pm} e^{ \pm i \omega_{I}\left(t-t^{\prime}\right)} .
$$

In Eq. (111), $N_{I}^{ \pm}$acquires different meanings depending on the interaction. In the e-p case it represents the occupation of the phonon bath at a given temperature that, for simplicity, we assume to be fixed. An extended derivation can be carried on by taking into account the simultaneous set of equations of motion for the electrons, phonons, and photons. However, here we would like to outline the method so we use as test case the electronic motion only.

In the e-p and e- $\gamma$ cases, then, $N_{I}^{+}=N_{I}(\beta)+1$ and $N_{I}^{-}=N_{I}(\beta)$ with $N_{I}(\beta)$ that Bose occupation and $\beta=1 / k_{B} T_{e l}$ with $k_{B}$ the Boltzmann constant and $T_{e l}$ the lattice temperature. In the e-e case $N_{I}^{+}=\rho_{k k}(t)\left[1-\rho_{l l}(t)\right]$ and $N_{I}^{-}=\rho_{l l}(t)\left[1-\rho_{k k}(t)\right]$.

By using Eq. (111) in Eq. (107), we get that

$$
\Sigma_{i j}^{\lessgtr}\left(t, t^{\prime}\right)=i \sum_{I}\left[\left(R^{I}\right)^{\dagger} G^{\lessgtr}\left(t, t^{\prime}\right) R^{I}\right]_{i j} W_{I}^{\lessgtr}\left(t-t^{\prime}\right) .
$$

\section{A. Generalized Baym-Kadanoff ansatz}

Now that $W \lessgtr$ is a function of the time difference, in order to close Eq. (99) in the space of the single-time density matrices we introduce the generalised Baym-Kadanoff ansatz. The GBKA is an ansatz for $G^{\lessgtr}$ which turns it, and hence the collision integral, into a functional of $\rho$ and $G^{r / a}$ :

$$
\begin{aligned}
& G^{<}\left(t, t^{\prime}\right)=-G^{r}\left(t-t^{\prime}\right) \rho\left(t^{\prime}\right)+\rho(t) G^{a}\left(t-t^{\prime}\right), \\
& G^{>}\left(t, t^{\prime}\right)=+G^{r}\left(t-t^{\prime}\right) \bar{\rho}\left(t^{\prime}\right)-\bar{\rho}(t) G^{a}\left(t-t^{\prime}\right),
\end{aligned}
$$

where $\bar{\rho}=1-\rho$. To transform $S^{\mathrm{dyn}}(t)$ into a functional of the density matrix, and hence to close Eq. (99), one needs to express the propagator $G^{r}$ in terms of $\rho$. Depending on the system there are optimal approximations to the propagator, the most common one being the quasiparticle (QP) propagator

$$
G^{r}\left(t, t^{\prime}\right)=-i \theta\left(t-t^{\prime}\right) T e^{-i \int_{t^{\prime}}^{t} d \bar{t} h^{\mathrm{QP}}(\bar{t})} .
$$

For (small) finite systems the choice $h^{\mathrm{QP}}=h^{\text {eq }}$ (usually $h^{\text {eq }}$ is the HF single-particle Hamiltonian) is a good choice. For extended systems, however, the lack of damping in $h^{\text {eq }}$ prevents the system to relax. In these cases the propagator is typically corrected by adding non-Hermitian terms given by the quasiparticle lifetimes $h^{\mathrm{QP}}=h^{\mathrm{eq}}+i \gamma[31,38,56-58]$.

By using Eq. (112) we rewrite $S^{\text {dyn }}(t)$ as

$$
\begin{aligned}
S_{i j}^{\mathrm{dyn}}[\{G\},\{\Sigma\}](T) \equiv & S_{i j}^{\mathrm{dyn}}[\{G\}](T)=i \sum_{I} \int_{-\infty}^{T} d t\left\{\left[\left(R^{I}\right)^{\dagger} G^{>}\left(T, t^{\prime}\right) R^{I} G^{<}\left(t^{\prime}, T\right)\right]_{i j} W_{I}^{>}\left(T-t^{\prime}\right)\right. \\
& \left.+\left[G^{<}\left(T, t^{\prime}\right)\left(R^{I}\right)^{\dagger} G^{>}\left(t^{\prime}, T\right) R^{I}\right]_{i j} W_{I}^{>}\left(t^{\prime}-T\right)-(\lessgtr \rightleftarrows \gtrless)\right\},
\end{aligned}
$$

and, finally, we use Eqs. (113) and $\left(113^{\prime}\right)$ to rewrite the first terms of Eq. (115):

$$
\left[\left(R^{I}\right)^{\dagger} G^{>}\left(T, t^{\prime}\right) R^{I} G^{<}\left(t^{\prime}, T\right)\right]_{i j} W_{I}^{>}\left(T-t^{\prime}\right)=(-i) \sum_{ \pm}\left[\left(R^{I}\right)^{\dagger} G^{r}\left(T-t^{\prime}\right) \bar{\rho}\left(t^{\prime}\right) R^{I} \rho\left(t^{\prime}\right) G^{a}\left(t^{\prime}-T\right)\right]_{i j} N_{I}^{ \pm} e^{ \pm \omega_{I}\left(T-t^{\prime}\right)}
$$

with $\bar{\rho}_{i j}(T) \equiv \delta_{i j}-\rho_{i j}(T)$. We now notice that, because of the time ordering of $G^{(r / a)}$, the integral in Eq. (115) runs between $-\infty$ and $T$.

In order to proceed with the derivation and obtain a final expression that can be easily compared with the well-known Boltzmann equation, we use a drastic but simple approximation for $G^{(r / a)}$ based on the noninteracting approximation

$$
G_{i j}^{r}(T) \approx-i \theta(T) e^{-i \varepsilon_{i} T} \delta_{i j}
$$

Equation (117) allows us to carry on to the final steps of the derivation in a more comfortable way by introducing the scattering matrix $S_{i j}^{I s}$ as

$$
S_{i j}^{I s}(T) \equiv-i \int_{-\infty}^{T} d t\left[e^{-i s \omega_{I} t}\left(R_{k i}^{I}\right)^{*} e^{i\left(\varepsilon_{j}-\varepsilon_{k}\right)(T-t)}\right] \underbrace{\left[\bar{\rho}_{k l}(t) R_{l n}^{I} \rho_{n j}(t) N_{I}^{-s}-\rho_{k l}(t) R_{l n}^{I} \bar{\rho}_{n j}(t) N_{I}^{s}\right]}_{\xi_{k j}^{I s}(t)} .
$$

In Eq. (118), we have also defined the $\xi_{k j}^{I S}(t)$ function. The $S^{\text {dyn }}(t)$ can be finally rewritten in terms of $S$ as

$$
S_{i j}^{\mathrm{dyn}}(T) \equiv i \sum_{I, \pm}\left[e^{i s \omega_{I} t} S_{i j}^{I s}(T)+\text { H.c. }\right] .
$$




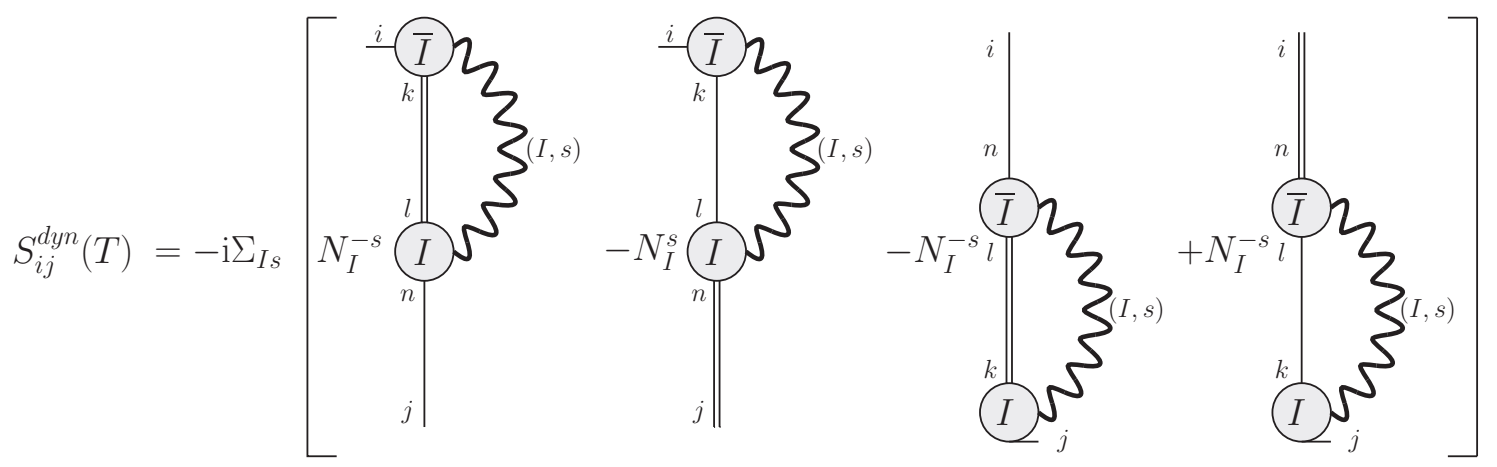

FIG. 7. Graphical representation of Eq. (121). A single line represents the density matrix $\rho$, while the double line is its adjoint $\bar{\rho}$. The wiggled line, in the spirit of Fig. 6, represents a generic interaction propagator.

\section{B. Completed collision approximation}

The time integral in Eq. (118) can be removed analytically by using the well-known completed collision approximation. This is based on the adiabatic ansatz introduced in Ref. [39] which is based on the assumption that the characteristic time scales of the dynamics are much longer compared to the time window where the physical properties are calculated. We will discuss more in detail the adiabatic ansatz in the next sections. Here, we just formulate it by approximating $\xi_{k j}^{I S}(t) \approx \xi_{k j}^{I S}(t)$ in Eq. (118), so to take it outside the time integral. At this point, this can be solved analytically leading to the final expression for the $S$ function:

$$
S_{i j}^{I s}(T) \approx(-i)\left(R_{k i}^{I}\right)^{*} \frac{e^{-i s \omega_{I} T}}{\varepsilon_{k}-\varepsilon_{j}-s \omega_{I}+i 0^{+}} \xi_{k j}^{I s}(T) .
$$

By plugging Eq. (120) into (119) we get the final, explicit, form of $S^{\mathrm{dyn}}(T)$ :

$$
S_{i j}^{\mathrm{dyn}}(T)=-i \sum_{I s}\left[\frac{\left(R_{k i}^{I}\right)^{*} \bar{\rho}_{k l}(T) R_{l n}^{I} \rho_{n j}(T) N_{I}^{-s}-\left(R_{i k}^{I}\right)^{*} \rho_{k l}(T) R_{l n}^{I} \bar{\rho}_{n j}(T) N_{I}^{s}}{\left(\varepsilon_{k}-\varepsilon_{j}-s \omega_{I}-i 0^{+}\right)}-\text {H.c. }\right] \text {. }
$$

Equation (121) represents an important result of this work. It shows that for any kind of interaction (e-e, e-p, e- $\gamma$ ) the scattering term of the equation of motion for the density matrix can be rewritten in a closed form.

Now, before moving to the next section where we will turn Eq. (121) into a working scheme to calculate several quantities, we want to analyze in detail the structure of $S^{\text {dyn }}$ in order to draw its very general properties. Indeed, in simple terms, Eq. (121) represents an elemental scattering event, where an initial state $(i)$ is scattered in a series of states $[(k),(l),(n)$, and finally $(j)]$ via the emission/absorption of an elemental excitation (photon, e-h pair, phonon).

In order to see this graphically, we proceed as follows. We associate a density matrix $\rho_{i j}(T)$ with a single line connecting the state $j$ to the state $i$, while $\bar{\rho}_{i j}(T)$ is represented by a double line. A large circle $(\bigcirc)$ will represent a generic interaction matrix $R^{I}$. A label $\bar{I}$ will denote an adjoint matrix $\left(R^{I}\right)^{\dagger}$. A wiggled line, instead, represents the propagation of an excitation $(I, s)$. By using these simple rules, the generic form of $S_{i j}^{\mathrm{dyn}}(T)$ is shown in Fig. 7.

Now, the next step is to link the properties of $S^{\mathrm{dyn}}(T)$ to the actual quantities that are measured in a typical pump-and-probe experiment. The procedure that reduces the complex dynamical equation to the simple Eq. (121) form is schematically represented in Fig. 8.

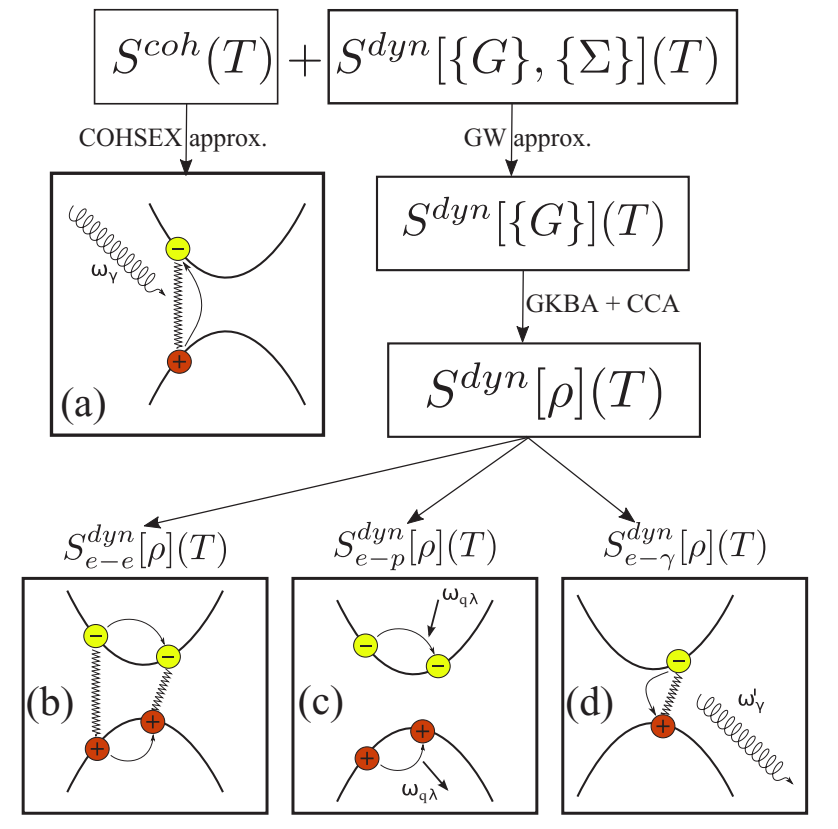

FIG. 8. Schematic representation of the different links between the terms of Eq. (123) and the most elemental physical processes occurring in a typical pump-and-probe experiment: the photoexcitation (a), the relaxation via e-e scattering (b), the relaxation and dissipation via e-p scattering (c), and the final, slow, radiative recombination (d). It is crucial to note that the use of $\Sigma_{\text {COHSEX }}$ allows to include excitonic effects (caused by the electron-hole attraction) in all the processes. 


\section{CARRIER DYNAMICS, TRANSIENT ABSORPTION, AND LIGHT EMISSION IN THE ADIABATIC REGIME}

In the typical experiment sketched in Fig. 1, a strong pump laser field is followed by a second weaker probe impulse whose physical properties are measured as a function of the pump-probe temporal delay. The low intensity of the probe and pump field and their temporal duration and delay can be used to simplify further the equations. Those are the physical basis for the low-intensity approximation and for the introduction of the adiabatic ansatz regime. These two special regimes are motivated by well-defined physical arguments that are introduced in this section.

The aim of this section is to use the fact that Eq. (121) is closed in the space of the density matrices and rewrite all possible observables relevant to the dynamics schematically represented in Fig. 1 as functions of the time-dependent density matrix.

\section{A. Adiabatic ansatz}

The adiabatic ansatz has been first introduced in Ref. [39] and in the following we present a short review in order to introduce the physical basis for the low-intensity and completed collision approximations. The temporal geometry of the pump and probe fields must appear explicitly in Eq. (19) connected to two components of the external charge

$$
\rho_{\text {ext }}(1)=\rho_{\text {ext }}^{\mathrm{p}}(1)+\rho_{\text {ext }}^{\mathrm{P}}(1)
$$

with $\rho^{\mathrm{P} / \mathrm{p}}$ referring to, respectively, the pump/probe components of the external charge. These terms define the pump and probe fields $\mathbf{E}^{\mathrm{P} / \mathrm{p}}(1)=-\nabla \phi_{\mathrm{ext}}^{\mathrm{P} / \mathrm{p}}(1)$. In a pump and probe experiment we have that, in general, $\left|\mathbf{E}^{\mathrm{P}}\right| \gg\left|\mathbf{E}^{\mathrm{p}}\right|$ and the two fields are separated by a temporal delay $\tau$.

The delay time $\tau$ is a crucial ingredient of the dynamics. Indeed, the pump field excitation induces several processes with different time scales. The laser excitation induces off-diagonal matrix elements of the density matrix $\rho_{i j}$, which in turn corresponds to a laser-induced current $\mathbf{J}(1)=\sum_{i j} \mathbf{j}_{i j}\left(\mathbf{r}_{1}\right) \rho_{j i}\left(t_{1}\right)$, with $\mathbf{j}_{i j}$ the matrix elements of Eq. (7). The time scale which describes the decay of the polarization and of the current $\tau_{\text {pol }}$ is dictated by relaxation processes.

At the same time, the pump excites electrons from the valence to the conduction bands and these carriers first relax towards the band minimum (in the case of electrons) and band maximum (in the case of holes). This relaxation occurs on a time scale $\tau_{\text {carr }}$. Thus, after a time $\tau_{\max }=\max \left(\tau_{\text {pol }}, \tau_{\text {carr }}\right)$, we may say that the system is in a quasistationary state. In this regime, the time to relax back to the ground state is dictated by e- $\gamma$ scattering and can be of the order of picoseconds.

The key assumption of the adiabatic ansatz is that the delay $\tau$ is chosen in such a way that, for times $t \approx \tau$, we have that $\rho(t+\Delta t) \approx \rho(t)$, if $\Delta t \ll \tau_{\max }$. The fundamental idea of such approximation is that, during the measurement process, the nonequilibrium configuration of the system is frozen.

By using Eq. (122) in the external interaction term of $h$, we can now make explicit the different terms contributing to Eq. (99). We start by using for the coherent part the COHSEX approximation, and by splitting $S^{\mathrm{dyn}}$ in the three terms induced by the different possible interactions

$$
\begin{aligned}
& \frac{d}{d T} \rho(T)+i\left[h_{\mathrm{ext}}(T), \rho(T)\right] \\
& \quad=-S^{\mathrm{coh}}[\rho](T)-S_{\mathrm{e}-\mathrm{e}}^{\mathrm{dyn}}[\rho](T)-S_{\mathrm{e}-\mathrm{p}}^{\mathrm{dyn}}[\rho](T)-S_{\mathrm{e}-\gamma}^{\mathrm{dyn}}[\rho](T) .
\end{aligned}
$$

The physical contents of the above equations are made clear by Eq. (121). In all the three cases the elemental process described by $S^{\text {dyn }}$ is a single transition from the electronic state $i$ to the electronic state $j$ by spanning all possible intermediate states composed by an electron in the level $k$ and a plasma (e-e case), phonon (e-p case), and photon (e- $\gamma$ ) excitation. The fact that it is a single transition is a consequence of the use of a $G W$ approximation.

We start by noticing that, when the external pump and probe fields are zero, $\rho$ is diagonal:

$$
\left.\rho_{i j}(T)\right|_{\mathbf{E}^{\mathrm{P}}=\mathbf{E}^{\mathrm{p}}=\mathbf{0}}=\delta_{i j} f_{i} .
$$

Equation (123) is a nonlinear equation whose nonlinearity is driven by the pump field. This nonlinearity mixes the diagonal and off-diagonal components of $\rho$ creating a complex interplay between the induced carrier occupations and the related polarization. However, in the case of a low-intense pumping field, if the system has reached the quasistationary state, we can approximate in the right-hand side of Eq. (121)

$$
\begin{aligned}
& \rho_{k l}(T) \approx \delta_{k l} f_{k}(T), \\
& \bar{\rho}_{k l}(T) \approx \delta_{k l}\left[1-f_{k}(T)\right] .
\end{aligned}
$$

We will refer to this set of approximations as low-intensity approximation (LIA). This is a well-established physical regime used in a wealth of experimental setups where the density of carriers created in the conduction bands is low enough to not alter substantially the physical properties of the material.

\section{B. Carrier dynamics}

In the LIA, the complex structure of the dynamical kernel can be further reduced to a compact and simple form

$$
\begin{aligned}
S_{i j}^{\mathrm{dyn}}(T) \approx & \left.S_{i j}^{\mathrm{dyn}}(T)\right|_{\text {LIA }} \\
= & i \sum_{n}\left[\gamma_{i j n}^{(-)}(T) \rho_{n j}(T)+\rho_{i n}(T) \gamma_{n i j}^{(+)}(T)\right. \\
& \left.-\tilde{\gamma}_{i j n}^{(-)}(T) \bar{\rho}_{n j}(T)-\bar{\rho}_{i n}(T) \tilde{\gamma}_{n i j}^{(+)}(T)\right],
\end{aligned}
$$

with

$$
\begin{aligned}
& \gamma_{i j n}^{( \pm)}(T)=(-i) \sum_{I s} \frac{\left(R_{k i}^{I}\right)^{*} f_{k}(T) R_{k n}^{I} N_{I}^{-s}}{\left(\varepsilon_{k}-\varepsilon_{j}-s \omega_{I} \pm i 0^{+}\right)}, \\
& \tilde{\gamma}_{i j n}^{( \pm)}(T)=(-i) \sum_{I s} \frac{\left(R_{k i}^{I}\right)^{*}\left[1-f_{k}(T)\right] R_{k n}^{I} N_{I}^{s}}{\left(\varepsilon_{k}-\varepsilon_{j}-s \omega_{I} \pm i 0^{+}\right)} .
\end{aligned}
$$

In obtaining Eqs. (126) and (127), we have used the LIA only on the internal density matrices as their indexes are free and do not impose the $S^{\text {dyn }}$ to be diagonal.

We can now link Eq. (123) to the complex dynamics we were aiming at describing at the beginning of this work, schematically represented in Fig. 1. Physically, the different 
contributions to $S^{\text {dyn }}$ represent the different channels that concur to the dynamics following the primary pump excitation. To see in practice their effect, we move to the splitting of Eq. (123) in carrier and polarization dynamics.

The equation of motion for the carrier occupations is readily obtained by taking the diagonal components of the $\rho$ solution of Eq. (123). In this case, the diagonal components of $S^{\text {dyn }}$ acquire a simple interpretation. Indeed, from Eqs. (126), (127), and $\left(127^{\prime}\right)$ it immediately follows that

$$
\begin{aligned}
\left.S_{i i}^{\mathrm{dyn}}(T)\right|_{\text {LIA }}= & 2 \pi \sum_{I s k}\left|R_{k i}^{I}\right|^{2}\left\{\left[1-f_{k}(T)\right]\right. \\
& \times f_{i}(T) \delta\left(\varepsilon_{i}-\varepsilon_{k}-s \omega_{I}\right) N_{I}^{-s} \\
& \left.-f_{k}(T)\left[1-f_{i}(T)\right] \delta\left(\varepsilon_{i}-\varepsilon_{k}-s \omega_{I}\right) N_{I}^{s}\right\} \\
= & \sum_{I} \gamma_{i}^{(I, \mathrm{e})}(T) \rho_{i i}(T)-\gamma_{i}^{(I, \mathrm{~h})}(T) \bar{\rho}_{i i}(T) .
\end{aligned}
$$

Equation (128) represents a generic Markovian scattering of the electron/hole [labeled by the $(\mathrm{e} / \mathrm{h})$ superscripts] in the state $i$ to the generic state $k$ mediated by the emission $(s=+1)$ or absorption $(s=-1)$ of a generic boson of energy $\omega_{I}$. In the e-e case, this boson is an additional electron-hole pair. In the $\mathrm{e}-\mathrm{p}$ case the boson is a phonon, and in the e- $\gamma$ channel it is a photon.

In the e-p and e-e cases, Eq. (128) reduces to the equation derived previously by one of us [38] and applied to the interpretation of the time-resolved two-photon photon-emission experiment of bulk silicon [20]. However, the present case extends the derivation to the e-p channel. This extension defines in a pure NEQ framework the radiative electron/hole lifetimes. At difference with the usual formulation [59] the $\gamma_{i}$ lifetimes are time dependent and depend on the time fluctuations of the carrier occupations. Moreover, by means of the presence of $S^{\mathrm{coh}}$, the coupling with the external laser field is correctly described.

\section{Light absorption}

Starting from the equilibrium condition, an external field will induce an electronic dipole defined as the expectation value of the dipole operator, defined in terms of the density matrix

$$
\hat{d}=\eta_{p} \cdot \hat{\mathbf{d}} \equiv \int d \mathbf{r}\left(\eta_{p} \cdot \mathbf{r}\right) \hat{\rho}(\mathbf{r}, \mathbf{r})=d_{i j} \hat{\rho}_{j i},
$$

with $d_{i j}=\int d \mathbf{r} \varphi_{i}^{*}(\mathbf{r})\left(\eta_{p} \cdot \mathbf{r}\right) \varphi_{j}(\mathbf{r})$ the dipole matrix elements. For simplicity, in Eq. (129), we have assumed that the pump and probe fields are polarized along the $\eta_{\mathbf{p}}$ direction. The time-dependent expectation value of the dipole operator $d(T)$ is then given by

$$
d(T)=\langle\Psi(T)|\hat{d}| \Psi(T)\rangle=d_{i j}\left\langle\Psi(T)\left|\hat{\rho}_{j i}\right| \Psi(T)\right\rangle,
$$

and can be calculated using the electronic polarization function $p_{\mathrm{e}}(1,2)$ as

$$
\begin{aligned}
\delta d(T) & =d_{i j} \int d t^{\prime} p_{j i, \mathrm{e}}\left(T, t^{\prime}\right) d_{k l} E_{p}\left(T^{\prime}\right) \\
& =\int d t^{\prime}\left[d \circ \chi\left(T, t^{\prime}\right) \circ d\right] E_{p}\left(T^{\prime}\right) .
\end{aligned}
$$

In Eq. (131) we used the conventions listed in the Appendix [see Eq. (A2)].

The absorption coefficient $\mathfrak{S}^{\tau}(\omega)$ can be easily calculated from the $\chi$ as [39]

$$
\mathfrak{S}^{\tau}(\omega)=-2 \omega|e(\omega)|^{2} \operatorname{Im}\left[d \circ \chi^{\tau}(\omega) \circ d\right],
$$

with $\chi^{\tau}(\omega)$ defined in Sec. VII and obtained from $\chi\left(t, t^{\prime}\right)$ by applying the adiabatic ansatz. The problem is now how to calculate $\chi$.

From Eq. (131) and the definition of the total (external plus induced) scalar field [Eq. (30)], we know that the matrix components of the reducible electronic polarization function can be written as

$$
\begin{aligned}
\chi_{j k}\left(t, t^{\prime}\right)= & -i \theta\left(t-t^{\prime}\right) \times\left\langle\Psi_{g}\right| \\
& \times\left[\hat{c}_{i H}^{\dagger}(t) \hat{c}_{j H}(t), \hat{c}_{k H}^{\dagger}\left(t^{\prime}\right) \hat{c}_{l H}\left(t^{\prime}\right)\right]\left|\Psi_{g}\right\rangle,
\end{aligned}
$$

which, together with its irreducible counterpart $\tilde{\chi}$, can be rewritten as derivatives of the time-dependent density matrix

$$
\begin{aligned}
& \chi_{l k}^{j i}\left(t, t^{\prime}\right)=\frac{\delta \rho_{i j}(t)}{\delta U_{k l}\left(t^{\prime}\right)}, \\
& \tilde{\chi}_{j i}\left(t, t^{\prime}\right)=\frac{\delta \rho_{i j}(t)}{\delta \phi_{k l}^{\operatorname{ext}}\left(t^{\prime}\right)} .
\end{aligned}
$$

The derivation of the equation of motion for both $\chi$ and $\tilde{\chi}$ is obtained by applying the functional derivatives to the equation of motion for $\rho(T)$ :

$$
\begin{aligned}
\frac{d}{d t}\left\{\frac{\delta \rho_{j i}(t)}{\delta \phi_{k l}^{\mathrm{ext}}\left(t^{\prime}\right)}\right\}= & \frac{\delta}{\delta \phi_{k l}^{\mathrm{ext}}\left(t^{\prime}\right)}\left\{-i\left[h_{\mathrm{ext}}(t), \rho(t)\right]_{j i}\right. \\
& \left.-S_{j i}^{\mathrm{coh}}[\rho](t)-S_{j i}^{\mathrm{dyn}}[\rho](t)\right\} .
\end{aligned}
$$

The first term on the right-hand side of Eq. (135) is

$$
\begin{aligned}
\frac{\delta}{\delta \phi_{k l}^{\mathrm{ext}}\left(t^{\prime}\right)}\left\{\left[h_{\mathrm{ext}}(t), \rho(t)\right]_{j i}\right\}= & {\left[\delta_{j k} \rho_{l i}(t)-\rho_{j k}(t) \delta_{l i}\right] \delta\left(t-t^{\prime}\right) } \\
& +\left[h_{\mathrm{ext}}(t), \tilde{\chi}\left(t, t^{\prime}\right)\right]_{j i},
\end{aligned}
$$

where we have used the compact form given by Eq. (A4) to write the last term of Eq. (136). The derivative of $S^{\text {coh }}$ can be evaluated within the COHSEX approximation for $\Sigma_{s}$ [Eq. (105)]:

$$
\frac{\delta S_{j i}^{\mathrm{coh}}(t)}{\delta \phi_{k l}^{\mathrm{ext}}\left(t^{\prime}\right)}=i\left[\frac{\delta \Sigma^{s}(t)}{\delta \phi_{k l}^{\mathrm{ext}}\left(t^{\prime}\right)}, \rho(t)\right]_{j i}=i\left[K_{s} \circ \tilde{\chi}\left(t, t^{\prime}\right), \rho(t)\right]_{k l}
$$

In order to evaluate the functional derivative acting on the $S^{\text {dyn }}$ functions, we use the following chain rule:

$$
\frac{\delta}{\delta \phi_{k l}^{\mathrm{ext}}\left(t^{\prime}\right)}=\int d \bar{t} \frac{\delta \rho_{m n}(\bar{t})}{\delta \phi_{k l}^{\mathrm{ext}}\left(t^{\prime}\right)} \frac{\delta}{\delta \rho_{m n}(\bar{t})},
$$

so that

$$
\frac{\delta}{\delta \phi_{k l}^{\mathrm{ext}}\left(t^{\prime}\right)} S_{j i}^{\mathrm{dyn}}[\rho](t)=\int d \bar{t}\left[K^{\mathrm{dyn}}(t, \bar{t}) \circ \tilde{\chi}\left(\bar{t}, t^{\prime}\right)\right]_{j i} .
$$


Thus, the final equation for the longitudinal two-times linear response function becomes

$$
\begin{aligned}
& \frac{d}{d t} \tilde{\chi}\left(t, t^{\prime}\right)+i\left[h_{\mathrm{ext}}(t), \tilde{\chi}\left(t, t^{\prime}\right)\right] \\
& \quad+i\left[K^{s} \circ \chi\left(t, t^{\prime}\right)+\mathbb{1} \delta\left(t-t^{\prime}\right), \rho(t)\right] \\
& =-\int d \bar{t}\left[K^{\mathrm{dyn}}(t, \bar{t}) \circ \tilde{\chi}\left(\bar{t}, t^{\prime}\right)\right] .
\end{aligned}
$$

As explained in Sec. VII, the nonlocal time dependence of the left-hand side of Eq. (140) can be simplified by using the adiabatic ansatz. The most complicated term remains the dynamical kernel $K^{\text {dyn }}$, that we will extensively discuss in Sec. VIE. Indeed, $K^{\text {dyn }}$ is a common ingredient of the equations describing both the light absorption and emission.

\section{Light emission}

Thanks to the quantization of the electromagnetic field, we can now derive a closed expression for the light-emission spectrum. As it will be clear shortly, the present formulation allows to introduce coherently the combined effects induced by the e-e and e-p coupling. This represents an important step forward compared to the state-of-the-art formulation and, more importantly, it allows an efficient merging with DFT.

In order to derive the expression for the light-emission spectrum in terms of the density matrix and use Eq. (123) to create a link with the density-matrix equation of motion, we use here the Poynting vector. In its Hermitian form, this vector is written as

$$
\begin{aligned}
\hat{\mathbf{S}}(1) & =\frac{c}{8 \pi}\left[\hat{\mathbf{E}}^{\dagger}(1) \times \hat{\mathbf{B}}(1)-\hat{\mathbf{B}}^{\dagger}(1) \times \hat{\mathbf{E}}(1)\right] \\
& =\left.\frac{c}{8 \pi}\left[\hat{\mathbf{E}}^{\dagger}(1) \times \hat{\mathbf{B}}(2)-\hat{\mathbf{B}}^{\dagger}(2) \times \hat{\mathbf{E}}(1)\right]\right|_{2=1} .
\end{aligned}
$$

The photoemitted spectrum $I(\omega)$ along the direction $\eta$ is obtained from the Fourier transformation of the macroscopic spatial average of the projection of the Poynting vector along $\eta, I(\omega) \propto\langle\hat{\mathbf{S}}(\mathbf{r}, \omega) \cdot \eta\rangle$.

We now take the relations between the fields and the vector potential

$$
\begin{aligned}
& \hat{\mathbf{E}}(1)=-\frac{1}{c} \frac{\partial \hat{\mathbf{A}}(1)}{\partial t_{1}}, \\
& \hat{\mathbf{B}}(1)=\nabla \times \hat{\mathbf{A}}(1) .
\end{aligned}
$$

By using Eqs. (142) and (142'), we can write explicitly the expression for the lesser and greater transverse photon propagator as

$$
\begin{aligned}
& \mathcal{D}_{\alpha \beta}^{<}(1,2)=\frac{1}{4 \pi i}\left[\left\langle\hat{A}_{\beta}(2) \hat{A}_{\alpha}(1)\right\rangle-\left\langle\hat{A}_{\alpha}(1)\right\rangle\left\langle\hat{A}_{\beta}(2)\right\rangle\right], \\
& \mathcal{D}_{\alpha \beta}^{>}(1,2)=\frac{1}{4 \pi i}\left[\left\langle\hat{A}_{\alpha}(1) \hat{A}_{\beta}(2)\right\rangle-\left\langle\hat{A}_{\alpha}(1)\right\rangle\left\langle\hat{A}_{\beta}(2)\right\rangle\right] .
\end{aligned}
$$

It is now possible to split the expectation value of the Poynting vector into two parts: a classical part which contains the information of the macroscopic effects

$$
\begin{aligned}
\left\langle\hat{S}_{\alpha}(1)\right\rangle_{\text {class }}= & \frac{1}{2} \sum_{\substack{\beta=1 \\
\beta \neq \alpha}}^{3} \frac{\partial\left\langle\hat{A}_{\beta}(1)\right\rangle}{\partial t_{1}} \\
& \times\left[\frac{\partial\left\langle\hat{A}_{\alpha}(2)\right\rangle}{\partial r_{2, \beta}}-\frac{\partial\left\langle\hat{A}_{\beta}(2)\right\rangle}{\partial r_{2, \alpha}}\right],
\end{aligned}
$$

and a contribution due solely to correlation effects

$$
\begin{aligned}
\left\langle\hat{S}_{\alpha}(1)\right\rangle_{\text {corr }}= & \frac{1}{2} \sum_{\substack{\beta=1 \\
\beta \neq \alpha}}^{3} \frac{\partial}{\partial t_{1}}\left\{\frac{\partial}{\partial r_{2, \beta}}\left[\mathcal{D}_{\beta \alpha}^{>}(1,2)+\mathcal{D}_{\beta \alpha}^{<}(1,2)\right]\right. \\
& \left.-\frac{\partial}{\partial r_{2, \alpha}}\left[\mathcal{D}_{\beta \beta}^{>}(1,2)+\mathcal{D}_{\beta \beta}^{<}(1,2)\right]\right\}\left.\right|_{2=1} .
\end{aligned}
$$

Equation (145) demonstrates that the evaluation of the lightemission spectrum is linked to the calculation of the lesser and greater transverse photon propagators that can be rewritten in terms of the advanced/retarded counterparts and the transverse response function [10]

$$
\overleftrightarrow{\mathcal{D}} \gtrless(1,2)=\overleftrightarrow{\mathcal{D}}^{r}(1,3) \overleftrightarrow{P} \gtrless(3,4) \overleftrightarrow{\mathcal{D}}^{a}(4,2)
$$

The problem of calculating the advanced and retarded photon propagators is itself a complicated issue. Here, we assume to be interested in systems where the renormalization of the photons can be neglected. This is the case of simple solids and molecules where the electronic polarization effects on the electromagnetic field can be assumed to be negligible. We start, then, from an independent-particle approximation for $\mathcal{D}^{a / r}$ :

$$
\begin{aligned}
& \mathcal{D}_{\alpha \beta}^{r}(\mathbf{r}, t)=-\frac{i c^{2}}{2} \sum_{I} \tau_{\alpha \beta}^{I}\left[\xi_{I}(\mathbf{r}) e^{-i \omega_{I} t}-\text { H.c. }\right] \theta(t), \\
& \mathcal{D}_{\alpha \beta}^{a}(\mathbf{r}, t)=\frac{i c^{2}}{2} \sum_{I} \tau_{\alpha \beta}^{I}\left[\xi_{I}(\mathbf{r}) e^{-i \omega_{I} t}-\text { H.c. }\right] \theta(-t) .
\end{aligned}
$$

In Eqs. (147) and $\left(147^{\prime}\right)$, we have introduced the single free photon wave function $\xi_{I}(\mathbf{r}) \equiv 2 \pi /\left(\Omega \omega_{\mathbf{q}+\mathbf{G}}\right) e^{i \mathbf{r} \cdot(\mathbf{q}+\mathbf{G})}$. We remind here the convention introduced in Sec. V to label the photon state as $I \equiv(\mathbf{q}, \mathbf{G})$. By using Eqs. (147) and $\left(147^{\prime}\right)$, we rewrite Eq. (145) only in terms of $P_{\alpha \beta}^{\gtrless}$ :

$$
\begin{aligned}
\mathcal{D}_{\alpha \beta}^{>}(1,2)+\mathcal{D}_{\alpha \beta}^{<}(1,2)= & \frac{c^{4}}{4} \sum_{\substack{I, J \\
\alpha_{1} \alpha_{2}}} \int d 3 d 4\left[\xi_{I}\left(\mathbf{r}_{1}-\mathbf{r}_{3}\right) e^{-i \omega_{I}\left(t_{1}-t_{3}\right)}-\text { H.c. }\right] \tau_{\alpha \alpha_{1}}^{I}\left[P_{\alpha_{1} \alpha_{2}}^{>}(3,4)+P_{\alpha_{1} \alpha_{2}}^{<}(3,4)\right] \tau_{\alpha_{2} \beta}^{J} \\
& \times\left[\xi_{J}\left(\mathbf{r}_{4}-\mathbf{r}_{2}\right) e^{-i \omega_{I}\left(t_{4}-t_{2}\right)}-\text { H.c. }\right] \theta\left(t_{1}-t_{3}\right) \theta\left(t_{2}-t_{4}\right) .
\end{aligned}
$$


Equation (148) reduces the calculation of the light-emission spectrum $I(\omega)$ to the evaluation of the equation of motion for $P_{\alpha \beta}^{\gtrless}(1,2)$. It is evident, then, that we can now follow a path quite similar to the longitudinal case by using a series of chain rules to close the equation of motion in an algebraic form. Indeed, we start by the $P_{\alpha \beta}^{\gtrless}(1,2)$ definition

$$
P_{\alpha \beta}\left(\mathbf{r}_{1}, t_{1} ; \mathbf{r}_{2}, t_{2}\right)=-\frac{4 \pi}{c} \frac{\delta\left\langle J_{\alpha}^{\mathrm{ind}}\left(\mathbf{r}_{1}, t_{1}\right)\right\rangle}{\delta\left\langle A_{\beta}\left(\mathbf{r}_{2}, t_{2}\right)\right\rangle},
$$

and by rewriting the induced current as

$$
\begin{aligned}
J_{\alpha}^{\text {ind }}(\mathbf{r}, t) & =\left.i \Pi_{\alpha}\left(\mathbf{r}, \mathbf{r}^{\prime}\right) G\left(\mathbf{r}, t ; \mathbf{r}, t^{\prime}\right)\right|_{\substack{\mathbf{r}^{\prime}=\mathbf{r} \\
t^{\prime}=t^{+}}} \\
& =-\left.\Pi_{\alpha}\left(\mathbf{r}, \mathbf{r}^{\prime}\right) \rho\left(\mathbf{r}, \mathbf{r}^{\prime} ; t\right)\right|_{\mathbf{r}^{\prime}=\mathbf{r} .}
\end{aligned}
$$

Then, by using Eq. (9) and expanding the density matrix in the single-particle basis, we can rewrite the induced current in terms of $\rho(t)$ :

$$
J_{\alpha}^{\mathrm{ind}}(\mathbf{r}, t)=-\sum_{i j} \Pi_{i j, \alpha}(\mathbf{r}) \rho_{j i}(t),
$$

where $\Pi_{i j, \alpha}(\mathbf{r})=\left.\Pi_{\alpha}\left(\mathbf{r}, \mathbf{r}^{\prime}\right) \phi_{i}^{*}(\mathbf{r}) \phi_{j}\left(\mathbf{r}^{\prime}\right)\right|_{\mathbf{r}^{\prime}=\mathbf{r}}$. We also expand the variations of $\left\langle A_{\alpha}(1)\right\rangle$ in the photon basis

$$
\delta\left\langle A_{\alpha}(\mathbf{r}, t)\right\rangle=\frac{1}{2} \sum_{I}\left[\xi_{I}(\mathbf{r}) \delta A_{I, \alpha}(t)+\text { H.c. }\right] .
$$

By using Eqs. (152) and (151), we can rewrite Eq. (149) as

$$
\begin{aligned}
- & \frac{4 \pi}{c} \sum_{i j} \Pi_{i j, \alpha}(\mathbf{r}) \delta \rho_{j i}(t) \\
& =\frac{1}{2} \sum_{I} \int d t^{\prime} d \mathbf{r}^{\prime} P_{\alpha \beta}\left(\mathbf{r}, t ; \mathbf{r}^{\prime}, t^{\prime}\right)\left[\xi_{I}(\mathbf{r}) \delta A_{I, \beta}(t)+\text { H.c. }\right] .
\end{aligned}
$$

We can now recast the functional derivative with respect to $\delta A$ as

$$
-\frac{4 \pi}{c} \sum_{i j} \Pi_{i j, \alpha}(\mathbf{r}) \frac{\delta \rho_{j i}(t)}{\delta A_{I, \beta\left(t^{\prime}\right)}}=\frac{1}{2} \int d \mathbf{r}^{\prime} P_{\alpha \beta}\left(\mathbf{r}, t ; \mathbf{r}^{\prime}, t^{\prime}\right) \xi_{I}\left(\mathbf{r}^{\prime}\right),
$$

and its adjoint

$$
\begin{aligned}
& -\frac{4 \pi}{c} \sum_{i j} \Pi_{i j, \alpha}(\mathbf{r}) \frac{\delta \rho_{j i}(t)}{\delta A_{I, \beta\left(t^{\prime}\right)}^{*}} \\
& =\frac{1}{2}\left[\int d \mathbf{r}^{\prime} P_{\alpha \beta}\left(\mathbf{r}, t ; \mathbf{r}^{\prime}, t^{\prime}\right) \xi_{I}\left(\mathbf{r}^{\prime}\right)\right]^{*} .
\end{aligned}
$$

At this point, we can define the $I$ th component of the transverse linear response function as

$$
\mathbf{P}_{i j}^{I}\left(t, t^{\prime}\right)=\frac{\delta \rho_{i j}(t)}{\delta \mathbf{A}_{I}\left(t^{\prime}\right)} .
$$

This means that we can use the same procedure as we did for the electronic polarization function $\chi$ [see, for example,
Eq. (135)] and write

$$
\begin{aligned}
\frac{\delta}{\delta \mathbf{A}_{I}\left(t^{\prime}\right)}\left[h_{\mathrm{ext}}(t), \rho(t)\right]_{j i}= & \delta\left(t-t^{\prime}\right)\left[\mathbf{p}^{I}, \rho(t)\right]_{j i} \\
& +\left[h_{\mathrm{ext}}(t), \frac{\delta \rho(t)}{\delta \mathbf{A}_{I}\left(t^{\prime}\right)}\right]_{j i},
\end{aligned}
$$

with

$$
\mathbf{p}_{i j}^{I}=-\frac{i}{c} \int d \mathbf{r} \phi_{j}^{*}(\mathbf{r}) \xi_{I}(\mathbf{r}) \nabla \phi_{j}(\mathbf{r}) .
$$

In Eq. (157), we have used the compact notation defined by Eq. (A4) in order to simplify the notation. The terms involving the functional derivatives of the COHSEX and dynamical kernels will follow the same procedure of the longitudinal case. In the case of the COHSEX kernel contribution, indeed, we have that

$$
\begin{aligned}
\frac{\delta S_{j i}^{\mathrm{coh}}(t)}{\delta \mathbf{A}_{I}\left(t^{\prime}\right)} & =\int d \bar{t} \frac{\delta \rho_{m n}(\bar{t})}{\delta \mathbf{A}_{I}\left(t^{\prime}\right)} \frac{\delta S_{j i}^{\mathrm{coh}}(t)}{\delta \rho_{m n}(\bar{t})} \\
& =\left[K_{s} \circ \frac{\delta \rho(t)}{\delta \mathbf{A}_{I}\left(t^{\prime}\right)}, \rho(r)\right]_{j i},
\end{aligned}
$$

and for the dynamical kernel we will write

$$
\frac{\delta S_{j i}^{\mathrm{dyn}}(t)}{\delta \mathbf{A}_{I}\left(t^{\prime}\right)}=\int d \bar{t}\left(K^{\mathrm{dyn}}(t, \bar{t}) \circ \frac{\delta \rho(\bar{t})}{\delta \mathbf{A}_{I}\left(t^{\prime}\right)}\right)_{j i} .
$$

We can now derive the final equation of motion for $\mathbf{P}_{i j}^{I}\left(t, t^{\prime}\right)$ in similarity with what we did for $\chi\left(t, t^{\prime}\right)$ :

$$
\begin{aligned}
\frac{d}{d t} \mathbf{P}^{I}\left(t, t^{\prime}\right)+i\left[h_{\mathrm{ext}}(t), \mathbf{P}^{I}\left(t, t^{\prime}\right)\right] \\
\quad+i\left[K_{s} \circ \mathbf{P}^{I}\left(t, t^{\prime}\right)+\delta\left(t-t^{\prime}\right) \mathbf{p}^{I}, \rho(t)\right] \\
=-K^{\mathrm{dyn}}(t) \circ \mathbf{P}^{I}\left(t, t^{\prime}\right) .
\end{aligned}
$$

Equation (161) represents another important result of this work. It derives a closed equation for the transverse response function and, in turn, it provides a sound scheme to calculate the photoluminescence spectrum. As this equation is derived in a scheme where e-e and e-p are included, this means that, physically, we have all essential ingredients of the dynamics. The carriers excited by the primary pump pulse will, then, relax, dissipate, and participate in bound electron-hole pairs before recombining and emitting light.

A final methodological remark is due. Equation (161) is for the response function defined in Eq. (154). An equivalent equation can be derived for its complex conjugate, defined by Eq. $\left.\left(154^{\prime}\right)\right)$. The only difference with Eq. (161) would be that the matrix elements $\mathbf{p}_{i j}^{I}$ must be replaced with $\mathbf{q}_{i j}^{I}=$ $-\frac{i}{c} \int d \mathbf{r} \phi_{j}^{*}(\mathbf{r}) \xi_{I}^{*}(\mathbf{r}) \nabla \phi_{j}(\mathbf{r})$.

\section{E. Dynamical two-particle kernel}

A common ingredient of Eqs. (163) and (161) is the dynamical kernel $K$, defined in Eq. (139) as $\frac{\delta S_{j i}^{\mathrm{dyn}}[\rho](t)}{\delta \rho_{m n}(\bar{t})}$. We have now all ingredients to analyze the physical contents of $K^{\mathrm{dyn}}$ and interpret its properties. The problem is now how to calculate this functional derivative and how to use the LIA.

If we look back into Eq. (121) and Fig. 7, we see how to evaluate the functional derivative of Eq. (139). The point 


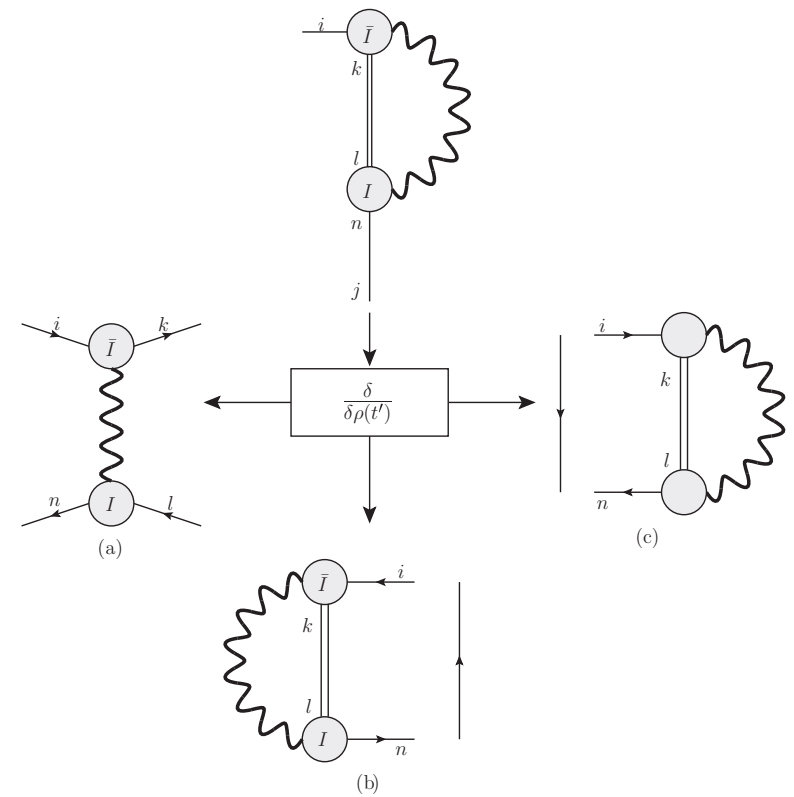

FIG. 9. Diagrammatic contributions to the dynamical kernel $K^{\text {dyn }}$ resulting from the application of the functional derivative to $S^{\mathrm{dyn}}$, within the $G W$ approximation.

is what happens if we apply the LIA. Indeed, we have two possibilities: to apply the LIA before doing the functional derivative or to apply it after the derivative.

As we will show shortly, this corresponds to neglect specific diagrams in the equation for the two-particle Green's function. In order to do so, let us follow a diagrammatic path. If we start from Fig. 7 we see that the derivative $\frac{\delta}{\delta \rho_{m n}(\bar{t})}$ can be applied both on $\rho$ and $\bar{\rho}$. Graphically this corresponds to open the diagram as schematically shown in Fig. 9.

We clearly see that two kinds of interactions contribute to the dynamical kernel: an electron-hole pair interaction [Fig. 9(a)], and a simple e-e [Fig. 9(b)] or hole-hole [Fig. 9(c)] interaction mediated by a generic boson, shown in the bottom right of Fig. 9. The electron-hole interaction is, in the equilibrium language, the well-known dynamical part of the screened electron-hole interaction. This has been studied in the framework of the BSE and showed to be connected and compensated with the dynamical self-energy effects $[60,61]$.

Now, if we apply the LIA before doing the functional derivative, we notice that the Fig. 9(a) disappears as it comes from the internal density matrix that, in Eq. (126) is approximated by its diagonal. Instead, if we apply the LIA after the functional derivative the two internal density matrices in Figs. 9(b) and 9(c) are approximated by occupation factors.

If we take the path of applying the LIA before doing the functional derivative, we get a closed expression for $K^{\mathrm{dyn}}$ :

$$
\begin{aligned}
K_{j i}^{\mathrm{dyn}}(t, \bar{t}) \approx & K_{j i}^{\mathrm{dyn}}(t) \delta(t-\bar{t}) \\
= & \delta(t-\bar{t})\left[\delta_{i l}\left(\gamma_{i l k}^{(-)}(t)+\tilde{\gamma}_{j l k}^{(-)}(t)\right)\right. \\
& +\delta_{j k}\left(\left(\gamma_{l k i}^{(+)}(t)+\tilde{\gamma}_{l k i}^{(+)}(t)\right)\right],
\end{aligned}
$$

which allows us to simplify the equation of motion for the longitudinal two-times response function, turning Eq. (140) into

$$
\begin{aligned}
\frac{d}{d t} \tilde{\chi}\left(t, t^{\prime}\right)+i\left[h_{\mathrm{ext}}(t), \tilde{\chi}\left(t, t^{\prime}\right)\right] \\
\quad+i\left[K^{s} \circ \tilde{\chi}\left(t, t^{\prime}\right)+\mathbb{1} \delta\left(t-t^{\prime}\right), \rho(t)\right] \\
=-K^{\mathrm{dyn}}(t) \circ \tilde{\chi}\left(t, t^{\prime}\right) .
\end{aligned}
$$

Equation (163) allows a simple and immediate physical interpretation as the $\gamma$ function simply representing a timedependent relaxation of the polarization that appears as a time-dependent broadening of the corresponding absorption peaks.

\section{THE FREQUENCY REPRESENTATION OF THE TRANSIENT ABSORPTION AND LUMINESCENCE SPECTRA}

In the equilibrium limit it is well known that the response function depends on the time difference and therefore it can be easily transformed in frequency space by applying a Fourier transform. This is a natural consequence of the time-translational invariance of the theory and reflects the fact the the energy of the system is conserved.

Out of equilibrium the energy of the electronic and nuclear subsystem is not conserved anymore as it flows back and forth to the electromagnetic field. As a consequence, both $\tilde{\chi}$ and $P$ are complex two-times functions. The next step is to use the adiabatic ansatz (see Sec. VI A) to change Eqs. (140) and (161) in order to have algebraic equations for $\tilde{\chi}$ and $P^{I, \alpha}$, as is in the state-of-the-art equilibrium case.

In the adiabatic ansatz, $\rho(t)$ and $A_{I, \alpha}(t)$ will change slowly in time. For times $\left(t, t^{\prime}\right) \approx \tau$ (with $\tau$ the pump-probe time delay) the response functions can be taken as a function of the relative time coordinate

$$
\begin{aligned}
\tilde{\chi}\left(t, t^{\prime}\right) & \approx \tilde{\chi}^{\tau}\left(t-t^{\prime}\right), \\
\mathbf{P}^{I}\left(t, t^{\prime}\right) & \approx \mathbf{P}^{I, \tau}\left(t-t^{\prime}\right) .
\end{aligned}
$$

Since in the adiabatic regime the density changes slowly in time, as long as the conditions described in Sec. VI A (a more detailed description can be found in Ref. [39]), the dynamic kernel of Eq. (140) can also be taken as a function of the relative time coordinate. Thus, we can finally rewrite Eq. (140) into an algebraic form for the frequency-dependent response function

$$
\begin{aligned}
& -i \omega \tilde{\chi}^{\tau}(\omega)+i\left[h_{\mathrm{ext}}(\tau), \tilde{\chi}^{\tau}(\omega)\right]+i\left[K_{s}^{\tau} \circ \tilde{\chi}^{\tau}(\omega)+1, \rho(\tau)\right] \\
& =-K^{\mathrm{dyn}, \tau}(\omega) \circ \tilde{\chi}^{\tau}(\omega) .
\end{aligned}
$$

The question now is how to bypass the calculation of the oneparticle density matrix $\rho(\tau)$ so that we can have an equation in which the only unknown quantity is the response function. In the equilibrium limit, we can always rotate the Hamiltonian $h_{\text {ext }}$ and the density into a basis where both would be diagonal, but this is not possible in nonequilibrium processes. Therefore, by following Ref. [39], we consider an orthogonal matrix $O(\tau)$ which rotates the Hamiltonian to the equilibrium basis and brings $h_{\text {ext }}(\tau)$ to its diagonal form

$$
\left[O^{\dagger}(\tau) h_{\mathrm{ext}}(\tau) O(\tau)\right]_{i j}=\delta_{i j} \epsilon_{i}(\tau)
$$


In this new basis, Eq. (166) reads as

$$
\begin{aligned}
& {\left[\omega-\Delta \epsilon(\tau)+i K^{\mathrm{dyn}, \tau}(\omega)\right] \circ \tilde{\chi}^{\tau}(\omega)} \\
& =\left[K_{s}^{\tau} \circ \tilde{\chi}(\omega)+1, \rho(\tau)\right] .
\end{aligned}
$$

Here, we have defined the energy tensor $\Delta \epsilon(\tau)_{i j}=$ $\left[\epsilon_{i}(\tau)-\epsilon_{j}(\tau)\right] 1_{i j}$. Following this definition we can write the $p q$

NEQ response function $\tilde{\chi}_{0}^{\tau}$ as

$$
\tilde{\chi}_{0}^{\tau}(\omega)=-\left[\omega-\Delta \epsilon(\tau)+i K^{\mathrm{dyn}, \tau}(\omega)\right]^{-1} \circ[\rho(\tau), 1] .
$$

If we now use the property

$$
\left[\rho(\tau), K_{s}^{\tau} \circ \tilde{\chi}^{\tau}(\omega)\right]=[\rho(\tau), 1] \circ K_{s}^{\tau} \circ \tilde{\chi}^{\tau}(\omega),
$$

we can finally obtain a Dyson-type equation for $\tilde{\chi}^{\tau}(\omega)$

$$
\tilde{\chi}^{\tau}(\omega)=\tilde{\chi}_{0}^{\tau}(\omega)+\tilde{\chi}_{0}^{\tau}(\omega) \circ K_{s}^{\tau} \circ \tilde{\chi}^{\tau}(\omega) .
$$

If we apply the same process to the transverse response function, we see that its differential equation (161) becomes

$$
\begin{aligned}
-i \omega \mathbf{P}^{I, \tau}(\omega)+i\left[h_{\mathrm{ext}}(\tau), \mathbf{P}^{I, \tau}(\omega)\right] \\
+i\left[K_{s}^{\tau} \circ \mathbf{P}^{I, \tau}(\omega)+\mathbf{p}^{I, \tau}, \rho(\tau)\right] \\
=-K^{\mathrm{dyn}, \tau}(\omega) \circ \mathbf{P}^{I, \tau}(\omega)
\end{aligned}
$$

and after reverting to the basis where $h_{\text {ext }}(\tau)$ is diagonal and following the same procedure we used to arrive at Eq. (171), we obtain the desired Dyson-type equation for $P^{I, \tau}(\omega)$ :

$$
\mathbf{P}^{I, \tau}(\omega)=\mathbf{P}_{0}^{I, \tau}(\omega)+\tilde{\chi}_{0}^{\tau}(\omega) \circ K_{s}^{\tau} \circ \mathbf{P}^{I, \tau}(\omega)
$$

with the NEQ $\mathbf{P}_{0}^{I, \tau}(\omega)$ being

$$
\mathbf{P}_{0}^{I, \tau}(\omega)=-\left[\omega-\Delta \epsilon(\tau)+i K^{\mathrm{dyn}, \tau}(\omega)\right]^{-1} \circ\left[\rho(\tau), \mathbf{p}^{I, \tau}\right] .
$$

Equation (174) represents another crucial result of this work. It provides, indeed, the basis for a fully ab initio implementation of the transient photoluminescence spectrum. This will allow to extend what has been already done previously [62] in the transient absorption case.

\section{MERGING WITH DENSITY-FUNCTIONAL THEORY}

In order to merge the BKE in the general DFT scheme, we follow the same strategy used in the standard MBPT approach [13]. This is based on the use of the Kohn-Sham (KS) Hamiltonian $h_{\mathrm{KS}}$ as the reference single-particle Hamiltonian. This means, in practice, that in Eq. (1) the $h$ operator is replaced by

$$
\begin{aligned}
h_{\mathrm{KS}} \phi_{i}(\mathbf{r}) & =\left[-\frac{\nabla^{2}}{2}+v_{\mathrm{ext}}(\mathbf{r})+v_{H}[\rho](\mathbf{r})+v_{\mathrm{xc}}[\rho](\mathbf{r})\right] \phi_{i}(\mathbf{r}) \\
& =\epsilon_{i}^{\mathrm{KS}} \phi_{i}(\mathbf{r}),
\end{aligned}
$$

with $v_{H}$ and $v_{\mathrm{xc}}$ the Hartree and the exchange-correlation potentials which, in DFT, are functional of the electronic density $\rho(\mathbf{r})$. Then, $\phi_{i}$ represents the basis of wave functions used in Eqs. (94) and (95).

DFT is a mean-field theory, where the electronic system is described by a group of pseudo-noninteracting particles which move under the influence of the $v_{\mathrm{xc}}$ potential that already contains some of the e-e correlation effects. This initial correlation already present in the KS Hamiltonian has several important consequences in the electronic [25] and also in the phononic dynamics [28].

As far as the electronic dynamics is concerned, $v_{\mathrm{xc}}$ can lead to subtle double-counting problems that are safely removed by defining, in Eq. (34),

$$
h_{\mathrm{ext}}=h_{\mathrm{KS}}-v_{\mathrm{xc}} \text {. }
$$

Another important ingredient of the present approach are the phonon modes. These, within the DFT scheme, are obtained by considering the total Hamiltonian $H$ as a functional of the atomic positions $\{\mathbf{R}\}$. The problem of finding the phonon modes reduces to the self-consistent calculation of derivatives of $H$. Indeed, if DFT is a self-consistent theory, DFPT [22,23] is its extension to take into account, self-consistently, the effect of static perturbations (like nuclear displacements). In this case, DFPT provides an exact description of phonons within the limits of a static and adiabatic approach.

Thus DFT and DFPT provide all ingredients of the present theory and allow a full ab initio implementation. Indeed, as discussed in Sec. V, we have that for each element of the theory we can define a DFT/DFPT counterpart. (a) As far as the single-particle electronic basis is concerned, the $\mathrm{KS}$ wave functions $\phi_{i}$ represent a natural definition. In this basis, all standard MBPT machinery can be used to calculate the ingredients of standard Hedin's equations [13]. (b) In the e-p case, phonons and e-p interaction matrix elements can be easily calculated within DFPT. Indeed, in this case, the ionic potential $V_{n}$ appearing in Eq. (108) is

$$
V_{n}(\mathbf{r}) \Rightarrow V_{s c f}(\mathbf{R}, \mathbf{r})=v_{H}(\mathbf{r})+v_{\mathrm{xc}}(\mathbf{r})-\sum_{\mathbf{R}} \frac{Z_{\mathbf{R}}}{|\mathbf{r}-\mathbf{R}|},
$$

and the phonon frequencies can be safely calculated within DFPT.

These simple connections demonstrate that, by simply implementing the NEGF framework in a KS basis, we can extend the predictive power of the many-body technique beyond the standard and well-known equilibrium limit.

\section{CONCLUSIONS}

In this work, we have carefully introduced, step by step, all mathematical and procedural steps needed to derive a simplified but accurate approach to the theory of out-of-equilibrium systems. We have started by the conclusions of previous works to integrate the electron-electron, electron-phonon, and electron-photon interactions in a consistent scheme based on the nonequilibrium Green's function theory.

We first derive a very general set of Hedin's equations where phonons and photons appear as the longitudinal and transverse components of the theory. We introduce, then, the corresponding longitudinal and transverse response and vertex functions showing how they are deeply connected. 
However, the complete theory appears clearly to be, by far, too complex to be implemented in realistic materials like nanostructures, surfaces, and all kind of systems currently within the range of applicability of DFT. As a consequence, in order to provide a workable scheme that can be potentially implemented in a DFT basis, we adopt the well-known $G W$ approximation that is extended to the out-of-equilibrium regime and to the transverse component of the self-energy.

This simplified form allows us to introduce a series of approximations: the generalized Baym-Kadanoff ansatz, the completed collision approximation, the low-intensity approximation, and the adiabatic ansatz. We discuss the physical motivations beyond these approximations and their range of applicability. By concentrating on the slow and weakly perturbed components of the dynamics we derive a set of closed equations for the carrier dynamics, transient absorption, and transient light emission written solely in terms of the time-dependent density matrix.

This represents a dramatic simplification of the scheme that can, now, be applied in a DFT framework to realistic materials. This is, indeed, the main conclusion of this work. The advanced and involved NEGF theory can be made simple enough to be applied in an $a b$ initio framework and provide the basis for a new set of tools available to the scientific community that we can group within the scopes a newly defined $a i$-NEGF approach.

The $a i$-NEGF extends the predictive power of the $a i$-MBPT approach to describe realistic systems out of equilibrium, thus opening the path to new applications, basic research, and numerical and methodological developments. This work represents, indeed, the formal proposal for such a working approach opening the path to many future developments and applications.

\section{ACKNOWLEDGMENTS}

We acknowledge financial support by the Futuro in Ricerca Grant No. RBFR12SW0J of the Italian Ministry of Education, University and Research, MIUR. A.M. also acknowledges the funding received from the European Union project MaX Materials design at the eXascale Project No. H2020-EINFRA-2015-1, Grant Agreement No. 676598, and Nanoscience Foundries and Fine Analysis-Europe Project No. H2020-INFRAIA-2014-2015, Grant Agreement No. 654360. P.M. thanks the Portuguese Foundation for Science and Technology (FCT) and the Portuguese Ministry for Education and Science, for the funding received through the Scholarship No. SFRH/BD/84032/2012 under the European Social Fund and the Programa Operacional Capital Humano (POCH).

\section{APPENDIX: CONVENTIONS FOR LINEAR ALGEBRA OPERATIONS}

In the derivation of the equations of Sec. VII we introduced the following ad hoc notation for the generic products of matrices:

$$
\begin{gathered}
(M \circ V)_{p q} \equiv M_{p q} V_{n m}, \\
(T \circ M \circ V) \equiv T_{p q} M_{q p} V_{n m}, \\
(M \circ N)_{m n} \equiv M_{m n} N_{q p}, \\
r s \\
{[N, V]_{m n}=-[V, N]_{m n} \equiv N_{m i} V_{i n}-V_{m i} N_{i n},} \\
p q \\
{[N, V]_{m n}=-[V, N]_{m n} \equiv N_{m i} V_{i n}-V_{m i} N_{i n} .}
\end{gathered}
$$

[1] R. Berera, R. van Grondelle, and J. T. M. Kennis, Photosynth. Res. 101, 105 (2009).

[2] G. Sansone, T. Pfeifer, K. Simeonidis, and A. I. Kuleff, ChemPhysChem 13, 661 (2012).

[3] J. Herrmann, M. Lucchini, S. Chen, M. Wu, A. Ludwig, L. Kasmi, K. J. Schafer, L. Gallmann, M. B. Gaarde, and U. Keller, New J. Phys. 17, 013007 (2015).

[4] A. I. Kuleff and L. S. Cederbaum, J. Phys. B: At., Mol. Opt. Phys. 47, 124002 (2014).

[5] S. M. Falke, C. A. Rozzi, D. Brida, M. Maiuri, M. Amato, E. Sommer, A. De Sio, A. Rubio, G. Cerullo, E. Molinari, and C. Lienau, Science 344, 1001 (2014).

[6] F. Rossi and T. Kuhn, Rev. Mod. Phys. 74, 895 (2002).

[7] F. Krausz and M. Ivanov, Rev. Mod. Phys. 81, 163 (2009).

[8] V. M. Axt and S. Mukamel, Rev. Mod. Phys. 70, 145 (1998).

[9] L. P. Kadanoff and G. Baym, Quantum Statistical Mechanics (W. A. Benjamin, New York, 1962).

[10] G. Pal, Y. Pavlyukh, W. Hübner, and H. C. Schneider, Eur. Phys. J. B 79, 327 (2011).

[11] G. Stefanucci and R. van Leeuwen, Nonequilibrium Many-Body Theory of Quantum Systems (Cambridge University Press, Cambridge, 2013).
[12] M. Bonitz, Quantum Kinetic Theory (B. G. Teubner, Stuttgart, 1998).

[13] G. Onida, L. Reining, and A. Rubio, Rev. Mod. Phys. 74, 601 (2002).

[14] C. Attaccalite, M. Grüning, and A. Marini, Phys. Rev. B 84, 245110 (2011).

[15] S. W. Koch, M. Kira, G. Khitrova, and H. M. Gibbs, Nat. Mater 5, 523 (2006).

[16] H. Shi, R. Yan, S. Bertolazzi, J. Brivio, B. Gao, A. Kis, D. Jena, H. G. Xing, and L. Huang, ACS Nano 7, 1072 (2013).

[17] G. B. Norris and K. K. Bajaj, Phys. Rev. B 26, 6706 (1982).

[18] J. Lefebvre and P. Finnie, Nano Lett. 8, 1890 (2008).

[19] T. Ichibayashi and K. Tanimura, Phys. Rev. Lett. 102, 087403 (2009).

[20] D. Sangalli and A. Marini, Europhys. Lett. 110, 47004 (2015).

[21] R. M.Dreizler and E. K. U. Gross, Density Functional Theory (Springer, Berlin, 1990).

[22] X. Gonze, Phys. Rev. A 52, 1096 (1995).

[23] S. Baroni, S. de Gironcoli, A. Dal Corso, and P. Giannozzi, Rev. Mod. Phys. 73, 515 (2001).

[24] M. van Schilfgaarde, T. Kotani, and S. Faleev, Phys. Rev. Lett. 96, 226402 (2006) 
[25] F. Aryasetiawan and O. Gunnarsson, Rep. Prog. Phys. 61, 237 (1998).

[26] P. Danielewicz, Ann. Phys. (NY) 152, 239 (1984).

[27] R. van Leeuwen, Phys. Rev. B 69, 115110 (2004).

[28] A. Marini, S. Poncé, and X. Gonze, Phys. Rev. B 91, 224310 (2015).

[29] S. Poncé, G. Antonius, Y. Gillet, P. Boulanger, J. Laflamme Janssen, A. Marini, M. Côté, and X. Gonze, Phys. Rev. B 90, 214304 (2014).

[30] E. Cannuccia and A. Marini, Phys. Rev. Lett. 107, 255501 (2011).

[31] A. Marini, Phys. Rev. Lett. 101, 106405 (2008).

[32] G. Antonius, S. Poncé, P. Boulanger, M. Côté, and X. Gonze, Phys. Rev. Lett. 112, 215501 (2014).

[33] F. Giustino, S. G. Louie, and M. L. Cohen, Phys. Rev. Lett. 105, 265501 (2010).

[34] M. Zacharias, C. E. Patrick, and F. Giustino, Phys. Rev. Lett. 115, 177401 (2015).

[35] P. B. Allen, Phys. Rev. Lett. 59, 1460 (1987).

[36] S. Das Sarma, J. K. Jain, and R. Jalabert, Phys. Rev. B 41, 3561 (1990).

[37] M. Bernardi, D. Vigil-Fowler, J. Lischner, J. B. Neaton, and S. G. Louie, Phys. Rev. Lett. 112, 257402 (2014).

[38] A. Marini, J. Phys.: Conf. Ser. 427, 012003 (2013).

[39] E. Perfetto, D. Sangalli, A. Marini, and G. Stefanucci, Phys. Rev. B 92, 205304 (2015).

[40] K. Henneberger and H. Haug, Phys. Rev. B 38, 9759 (1988).

[41] K. Hannewald, S. Glutsch, and F. Bechstedt, Phys. Rev. Lett. 86, 2451 (2001).

[42] M. F. Pereira and K. Henneberger, Phys. Rev. B 58, 2064 (1998).

[43] K. Hannewald, S. Glutsch, and F. Bechstedt, Phys. Rev. B 61, 10792 (2000).

[44] K. Hannewald, S. Glutsch, and F. Bechstedt, Phys. Rev. B 67, 233202 (2003).
[45] G. F. Bertsch, J. I. Iwata, A. Rubio, and K. Yabana, Phys. Rev. B 62, 7998 (2000).

[46] K. Yabana, T. Sugiyama, Y. Shinohara, T. Otobe, and G. F. Bertsch, Phys. Rev. B 85, 045134 (2012).

[47] C. Andrea Rozzi, S. Maria Falke, N. Spallanzani, A. Rubio, E. Molinari, D. Brida, M. Maiuri, G. Cerullo, H. Schramm, J. Christoffers, and C. Lienau, Nat. Commun. 4, 1602 (2013).

[48] K. Krieger, J. K. Dewhurst, P. Elliott, S. Sharma, and E. K. U. Gross, J. Chem. Theory Comput. 11, 4870 (2015).

[49] M. Ruggenthaler, J. Flick, C. Pellegrini, H. Appel, I. V. Tokatly, and A. Rubio, Phys. Rev. A 90, 012508 (2014).

[50] M. Bernardi, D. Vigil-Fowler, C. S. Ong, J. B. Neaton, and S. G. Louie, Proc. Natl. Acad. Sci. USA 112, 5291 (2015).

[51] M. F. Pereira and K. Henneberger, Phys. Rev. B 53, 16485 (1996).

[52] A. L. Fetter and J. D. Walecka, Quantum Theory of ManyParticles Systems (McGraw-Hill, New York, 1971).

[53] G. Strinati, H. J. Mattausch, and W. Hanke, Phys. Rev. B 25, 2867 (1982).

[54] L. Hedin and S. Lundqvist, Solid State Phys. 23, 1 (1970).

[55] G. Baym, Ann. Phys. (NY) 14, 1 (1961).

[56] H. Haug and C. Ell, Phys. Rev. B 46, 2126 (1992).

[57] M. Bonitz, D. Semkat, and H. Haug, Eur. Phys. J. B 9, 309 (1999).

[58] S. Latini, E. Perfetto, A.-M. Uimonen, R. van Leeuwen, and G. Stefanucci, Phys. Rev. B 89, 075306 (2014).

[59] M. Palummo, M. Bernardi, and J. C. Grossman, Nano Lett. 15, 2794 (2015).

[60] A. Marini and R. Del Sole, Phys. Rev. Lett. 91, 176402 (2003).

[61] F. Bechstedt, K. Tenelsen, B. Adolph, and R. Del Sole, Phys. Rev. Lett. 78, 1528 (1997).

[62] E. A. A. Pogna, M. Marsili, D. De Fazio, S. Dal Conte, C. Manzoni, D. Sangalli, D. Yoon, A. Lombardo, A. C. Ferrari, A. Marini, G. Cerullo, and D. Prezzi, ACS Nano 10, 1182 (2016). 